\title{
Covert Communication for Wireless Networks with Full-Duplex Multiantenna Relay
}

\author{
Ling Yang $\left(\mathbb{D},{ }^{1,2}\right.$ Weiwei Yang $\mathbb{D}^{1},{ }^{1}$ Liang Tang, ${ }^{1,3}$ Liwei Tao, ${ }^{1}$ Xingbo Lu, ${ }^{1}$ and Zhengyun $\mathrm{He}^{2}$ \\ ${ }^{1}$ College of Communications Engineering, PLA Army Engineering University, Nanjing 210 007, China \\ ${ }^{2}$ College of Railway Transportation, Hunan University of Technology, Zhuzhou 412 007, China \\ ${ }^{3}$ College of Science, Hunan University of Technology, Zhuzhou 412 007, China
}

Correspondence should be addressed to Weiwei Yang; weiweiyang1981@126.com

Received 19 August 2021; Accepted 22 November 2021; Published 7 January 2022

Academic Editor: Hou-Sheng Su

Copyright (C) 2022 Ling Yang et al. This is an open access article distributed under the Creative Commons Attribution License, which permits unrestricted use, distribution, and reproduction in any medium, provided the original work is properly cited.

In this work, we investigated a covert communication method in wireless networks, which is realized by multiantenna full-duplex single relay. In the first stage, the source node sends covert messages to the relay, and the relay uses a single antenna to send interference signals to the adversary node to protect the covert information being transmitted. In the second stage, the relay decodes and forwards the covert information received in the first stage; at the same time, the relay uses zero-forcing beamforming to send interference signals to the warden to ensure covert transmission. The detection error rate, transmission outage probability, maximum effective covert rate, and other performance indicators are derived in two stages, and the total performance of the system is derived and analyzed. Then, the performance indicators are verified and analyzed by simulation. Our analysis shows that the maximum effective covert rate of using the characteristics of multiantenna to interfere with Willie in the second stage is taken as the total covert performance of the system, and the transmission interruption probability is significantly less than that of the first stage, so the corresponding maximum effective concealment efficiency will be greater.

\section{Introduction}

1.1. Background. Networks are ubiquitous in nature and human society. Modern communication has been highly concerned by academic and industrial circles, resulting in many emerging sciences, such as the internet of things [1], complex networks [2-5], cognitive networks [6, 7], virtual reality, and augmented reality technology. Due to the broadcast characteristics of wireless channels, whether users are legal or not, researchers pay more and more attention to the security and privacy of information transmission. Taking full advantage of the uncertainty and unpredictability of wireless channels, physical layer security technology $[8,9]$ has become a mature technology, which has been applied to achieve secure transmission, focusing on information theory technology $[10,11]$. However, in many communication cases, not only the privacy and integrity of information need to be considered, but also the security of communication behavior needs to be protected, such as the existence of hidden communication [12]. In this context, covert communication $[13,14]$ emerged as a new security technology.

In 2013, B. A. Bash proved the reliable communication with low detection probability under additive white Gaussian noise (AWGN) channel theoretically for the first time [15] and proposed the square root rule of covert communication under AWGN channel. Then, the basic research of covert communication has attracted the interest of relevant scholars and has been studied in AWGN channels [16], discrete memoryless channels (DMC) $[17,18]$, and binary symmetric channel (BSC) [19]. At the same time, the basic limitations of covert communication under different channel conditions have been explored [20]. With the deepening of theoretical research, the concept of covert communication has gradually formed and developed. So far, researchers represent by Dr. B. A. Bash and Dr. Shihao Yan team of Macquarie University in Australia have made contributions to the basic theoretical research and performance analysis of covert communication technology; 
covert communication technology has attracted more and more researchers' attention, and its related research has gradually developed and enriched [21-23].

1.2. Previous Works and Motivations. Recently, more and more scenes and technologies about covert communication have been studied. In this paper, we focus on full duplex (FD), relay, and multiantenna covert technology.

Reference [24] explores the possibility and condition of covert communication in quasi-static wireless fading channel by using an FD receiver to transmit artificial noise (AN) to enhance covert performance. Reference [25] not only studies the influence of channel estimation inaccuracy and randomness on covert wireless transmission performance but also explores the effect of FD relay on covert performance. Furthermore, in ad hoc networks, the internet of things, and other random wireless networks, reference [26] also uses FD technology to effectively enhance the covert of communication.

Reference [25] also studies the influence of channel uncertainty of detecting attackers on covert wireless transmission performance in two-hop relay communication system. Reference [27] studies the covert and transmission reliability of covert wireless transmission schemes with the assistance of a single relay and shows that cooperative relay can enhance the performance of covert wireless transmission. In [28], a covert wireless transmission scheme is designed with the assistance of a wireless energy acquisition relay, and the expression of minimum error detection probability is derived. In [29], the problem of the covert wireless transmission assisted by the untrusted relay is studied. References $[30,31]$ study the covert wireless communication scheme with multihop relay transmission. In particular, reference [31] considers the problem of multihop covert relay transmission in unmanned aerial vehicle communication. Most of these researches focus on reducing transmission power by relay transmission to enhance covertness or improving the communication performance of legal links by using cooperative diversity gain. However, the probability of signal exposure caused by relay transmission increases, and the performance degradation is not clear.

From [32], an active eavesdropping scheme assisted by the covert pilot attack is designed for wireless monitoring scenarios, and covert wireless communication is realized by using the uncertainty of the detection channel. The communication process is divided into the channel estimation stage and the data transmission stage. Through the use of a malicious detection node (source node) equipped with multiple antennas to realize hidden pilot attacks on channel status information in the channel estimation stage, the information beam of the communication process is forced to point to the wireless monitoring node in the data transmission stage, and the probability of wireless monitoring success is improved. Reference [33] considers centralized and distributed antenna systems and discusses the coverage and reliability of transmission. In addition, the covert communication model of multiantenna detector is also studied in reference [34], and the influence of the increase of the number of detectors' antennas on the covert performance is discussed. In recent years, researchers consider using multiantenna jammers to improve the performance of covert communication systems [35]. In [36], the enhancement effect of multiantenna AN nodes on covert performance is studied, and it is pointed out that directional beamforming is the optimal AN strategy. In [14], an FD multiantenna receiver is used to achieve covert communication based on the uncertainty of interference power. The receiver first selects the best antenna to receive the covert information and then randomly selects one of the remaining antennas to generate $\mathrm{AN}$, resulting in the uncertainty of the detector so as to achieve covert communication.

In the present work, the FD, multiantenna and cooperative transmission method under covert communication needs to be further studied. On the one hand, the existing research of covert communication often focuses on the covert transmission of its own important information based on the relay forwarding the source's message and seldom considers that the information sent by the source are covert messages at the beginning, so the established scene has certain limitations. On the other hand, the reliability of relay nodes in covert wireless communications or covert wireless networks in the presence of eavesdropping is rarely involved. How to combine multiantenna, FD, cooperative technology, and covert communication is worthy of further study. Based on this, the research point of covert communication in multiantenna FD relay system is proposed.

1.3. Our Approach and Contributions. The main contributions of this paper are summarized as follows:

(i) We prove that the use of multiantenna FD relay is an effective way to achieve covert wireless communication in fading wireless channels. Multiantenna FD relay uses the advantages of multiantenna to design different antenna selection schemes in two stages to transmit signals with different power to cause Willie's confusion.

(ii) Based on the assumption of the Willie radiometer, we analytically derive Willie's optimal detection thresholds for the two stages of the system. When we define the optimal decision rules for the minimum detection error rate, the predetermined thresholds of the two stages are consistent, and the optimal detection performance is obtained with the minimum detection error probability.

(iii) For the given covert constraints, we give the design criteria of the optimal interference power of the first stage relay and the optimal transmission power of the second stage relay to forward the covert messages so as to maximize the optimal effective covert rate of the system.

(iv) Our analysis shows that for the same parameters, the transmission outage probabilities of the first stage, the second stage, and the total results have the same trend. Since the total maximum effective covert rate is smaller of the two stages, the total maximum effective covert rate has the same conclusion as that of the first stage. 
1.4. Organization. The rest of this paper is organized as follows. The system model is introduced in Section 2. Sections 3 and 4, respectively, introduce the two stages of the system. The first stage is that the source node sends covert messages to the relay, and the second stage is that the relay forwards covert messages to the destination node. The detection and covert performance of the system are deduced and analyzed in the two stages. In Section 5, the total performance of the system is studied. Section 6 provides the theoretical analyses are verified by numerical results. Finally, Section 7 describes some concluding remarks. A list of the fundamental variables is provided in Table 1 .

\section{System Model}

As shown in Figure 1, we consider a covert wireless communication model in a single relay multiantenna network, which includes a transmitter (Alice), a relay (Relay), a receiver (Bob), and an adversary (Willie). Among them, Relay is equipped with $N_{R}$ antennas, and other nodes are equipped with a single antenna. Since there is no direct communication link between Alice and Bob, it is necessary to help Alice send covert messages to Bob through Relay, while Willie tries to detect such covert transmissions, and each role knows each other's existence and location. Information transmission is divided into two stages. In the first stage, Alice sends covert messages to Relay, and Relay sends artificial noise $(A N)$ signals to Willie while receiving the messages. In order to improve the effective covert rate as much as possible, Relay selects the best antenna between it and Alice for receiving, one of the remaining $N_{R}-1$ antennas was selected at random to send AN to Willie. In the second stage, Relay selects an optimal antenna to forward Alice's signal to Bob by decode-and-forward and uses the remaining $N_{R}-1$ antennas to transmit AN signal to Willie by using zero-forcing beamforming $(Z F B)$.

Assuming that the transmission of the first stage is completed before the transmission of the second stage, it is necessary to ensure that neither stage can be detected by Willie in order to realize the covert transmission from Alice to Bob. Since Willie needs to detect whether Alice and Relay send covert messages in the first and second stages, respectively, considering the worse case, it is assumed that Willie knows $h_{A W}$ and $h_{R W}$ and that Relay knows $h_{A R}$, and since Relay knows Willie's existence, it is assumed that Relay also knows $h_{R W}$ [1]. The wireless channels are subject to independent quasi-static Rayleigh fading; the channels change independently between time slots and remain unchanged within the same communication time slot.

\section{The First Stage: Alice Transmits Covert Messages to Relay}

3.1. Relay Receives Covert Messages. The instantaneous signalto-interference-plus-noise ratio (SINR) at Relay is given by

$$
\gamma_{\text {Relay }}=\frac{P_{A} \max _{1 \leq i \leq N_{R}}\left(\left|h_{A R_{i}}\right|^{2}\right)}{\varphi P_{R_{j}}\left|h_{R_{j} R_{j}}\right|^{2}+\sigma_{R}^{2}} .
$$

Relay selects the best $i$-th antenna to receive the signal according to the channel conditions between Alice and Relay, and its channel coefficient is expressed as $h_{A R_{i}}$, where $i=1,2, \ldots, n, P_{A}$ is the power of Alice sending covert messages, $P_{R_{j}}$ is the power of AN sent by Relay, $P_{R_{j} R_{j}}$ is the channel coefficient of Relay itself, and $j$ is the $j$-th antenna randomly selected by Relay in $N_{R}-1$ antennas. Since Relay itself knows AN signal, the residual noise can be reconstructed and eliminated through self-interference elimination. $\phi$ is used to represent the self-interference elimination coefficient. $\phi=0$ is the ideal situation, while $0 \leq \phi \leq 1$ refers to different self-interference elimination levels [37]. $\sigma_{R}^{2}$ is the channel variance of Relay. The maximum power of Relay is $P_{R}^{\max }$. Assuming that the power is evenly distributed among the antennas of Relay, let $P_{R_{j}}^{\max }=P_{R}^{\max } / N_{R}$. We assume that $P_{A}$ is fixed [38], and both Relay and Willie know it. The $P_{R_{j}}$ changes from slot to slot, following a continuous uniform distribution over the interval $\left[0, P_{R_{j}}^{\max }\right]$, having a probability density function $(P D F)$ given by

$$
f_{P_{R_{j}}}(x)= \begin{cases}\frac{1}{P_{R_{j}}^{\max },} & 0 \leq x \leq P_{R_{j}}^{\max }, \\ 0, & \text { otherwise. }\end{cases}
$$

Reasons for setting $P_{R_{*}}$ to change between slots: the purpose of Relay to send $\mathrm{A}_{j} \mathrm{~N}$ power is to make the power received by Willie uncertain. Willie knows $h_{A W}$ in a time slot. If the AN power is constant, Willie can directly detect the covert transmission when Alice sends fixed covert messages, so the $P_{R_{j}}$ is not fixed.

In the first stage, when Alice sends covert messages, the signal received by Relay is as follows:

$$
\begin{aligned}
Y_{\text {Relay }}[i]= & \sqrt{P_{A}} \max \left(h_{A R_{i}}\right) x_{A}[i] \\
& +\sqrt{\varphi P_{R_{j}}} h_{R_{j} R_{j}} V_{R}[i]+n_{R}[i],
\end{aligned}
$$

where $x_{A}[i]$ represents the signals transmitted by Alice, satisfying $E\left[x_{A}[i] x_{A}^{\dagger}[i]\right]=1, i=1,2, \ldots, n$ represents the symbol index, $n$ is the total number of channels used in each slot, and $n_{R}[i]$ is the complex additive white Gaussian noise $(A W G N)$ at Relay with $\sigma_{R}^{2}$ as its variance, that is, $n_{R}[i] \sim C N\left(0, \sigma_{R}^{2}\right)$.

3.2. Detection Metrics at Willie. In the first stage, the SINR at Willie, in case Alice transmits, is given by

$$
\gamma_{\text {Willie1 }}=\frac{P_{A}\left|h_{A W}\right|^{2}}{P_{R_{j}}\left|h_{R_{j} W}\right|^{2}+\sigma_{W}^{2}},
$$

where $h_{A W}$ is the channel coefficient between Alice and Willie, $h_{R_{j} W}$ represents the channel coefficient between the $j$-th antenna that Relay randomly selects to send interference and Willie, $\sigma_{W}^{2}$ is the noise variance of Willie. In a communication time slot, Willie has to decide whether Alice has transmitted covert messages to Relay in the first stage. Therefore, Willie is faced with a binary hypothesis testing 
TABLE 1: List of fundamental variables.

\begin{tabular}{|c|c|}
\hline Symbol & Description \\
\hline SINR & Interference plus noise ratio \\
\hline$A W G N$ & The complex additive Gaussian noise \\
\hline$f(\cdot)$ & The cumulative distribution function \\
\hline$P_{X}$ & The power send by $X, X \in\{$ Alice, Relay, Bob, Willie $\}$ \\
\hline$y_{A}$ & The received signal at $A, A \in\{$ Bob, Relay, Willie $\}$ \\
\hline$h_{a, b}$ & The channel between $a$ and $b$ that both consist a single antenna \\
\hline$n_{A}[i]$ & The AWGN at $A, A \in\{$ Bob, Relay, Willie $\}$ \\
\hline$\gamma_{A}$ & The SINR on $A, A \in\{B o b$, Willie $\}$ \\
\hline$n$ & The number of each channel use \\
\hline$\sigma_{A}^{2}$ & The variance of $A W G N$ at node $A, A \in\{B o b$, Willie $\}$ \\
\hline$T_{W}^{A}$ & The average power received at Willie \\
\hline$\tau$ & The predefined detection threshold \\
\hline$\xi$ & The detection error rate \\
\hline$\tau^{*}$ & The optimal predefined detection threshold \\
\hline$\xi^{*}$ & The minimum detection error rate \\
\hline$\overline{R_{C}}$ & The maximized effective covert rate \\
\hline$F(\cdot)$ & The probability density function \\
\hline$\varepsilon$ & The predetermined covert communication condition value \\
\hline$R$ & The transmission rate \\
\hline$\delta$ & The transmission outage probability \\
\hline$N_{A}$ & Antenna number of $A, A \in\{$ Bob, Relay, Willie $\}$ \\
\hline$h_{a, b}$ & The channel vector between $a$ and $b$ consisting of one and multiple antennas, respectively \\
\hline$w_{a, b}$ & The received weighting vector on link $a-b$ \\
\hline$\dagger$ & The conjugate transpose operator \\
\hline$\|\bullet\|_{F}$ & The Frobenius norm \\
\hline$T^{\perp}$ & The projection idempotent matrix \\
\hline$\alpha$ & False alarm rate \\
\hline$\beta$ & Miss detection rate \\
\hline
\end{tabular}

problem, where the null hypothesis $H_{0}$ means that Alice has not sent covert messages, and the alternative hypothesis $H_{1}$ means that Alice has sent covert messages to Relay.

The signal received by Willie in the first stage can be expressed as follows:

$$
y_{\text {willie }}[i]= \begin{cases}\sqrt{P_{R_{j}}} h_{R_{j} W} v_{R}[i]+n_{W}[i], & H_{0}, \\ \sqrt{P_{A}} h_{A W} x_{A}[i]+\sqrt{P_{R_{j}}} h_{R_{j} W} v_{R}[i]+n_{W}[i], & H_{1},\end{cases}
$$

where $n_{W}[k]$ is the AWGN at Willie with $\sigma_{W}^{2}$ as its variance, that is, $n_{W}[k] \sim C N\left(0, \sigma_{W}^{2}\right)$. Willie does not know the value of $P_{R_{j}}$ in the time slot, but the value of $P_{A}$ is fixed and known. In the first stage, Willie attempts to detect whether $y_{\text {willie }}$ is $H_{0}$ or $H_{1}$. Through the application of Neyman-Perason criterion [39] and likelihood ratio test, the optimal decision rule for Willie to minimize his detection error is as follows:

$$
T_{W} \stackrel{D_{1}}{>} \underset{D_{0}}{>} \tau,
$$

where $T_{W}=1 / n \sum_{i=1}^{n} y_{W i l l i e 1}[i]$ is the average power received at Willie in the slot and $\tau$ is a predefined Willie's detection threshold. $D_{0}$ and $D_{1}$ are binary decisions that infer whether Alice sends covert messages. In this paper, we consider an

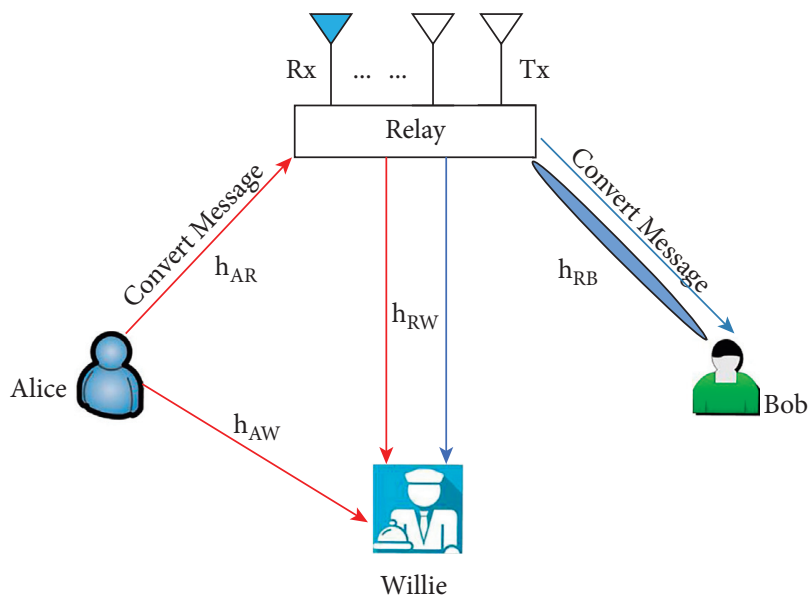

Phase 1

Phase 2:

Figure 1: System model.

infinite number of channel uses, that is, $n \longrightarrow \infty$. Therefore, we have

$$
T_{W}= \begin{cases}P_{R_{j}}\left|h_{R_{j} W}\right|^{2}+\sigma_{W}^{2}, & H_{0} \\ P_{A}\left|h_{A W}\right|^{2}+P_{R_{j}}\left|h_{R_{j} W}\right|^{2}+\sigma_{W}^{2}, & H_{1}\end{cases}
$$


At the end of the communication slot, Willie has to make a decision. The false alarm rate is defined as the probability of Willie making $D_{1}$ decision under condition $H_{0}$, which is expressed by $\alpha=P\left(D_{1} \mid H_{0}\right)$. Similarly, the miss detection rate is defined as the probability of Willie making $D_{0}$ decision under condition $H_{1}$, which is expressed by $\beta=P\left(D_{0} \mid H_{1}\right)$. Assuming that the prior probabilities of $H_{0}$ and $H_{1}$ are equal, Willie's detection performance can be judged by the detection error rate, which can be defined as follows:

$$
\xi \triangleq \alpha+\beta
$$

3.3. Detection Performance at Willie. According to Theorems 1 and 2 in [37], Willie's optimal detection threshold, minimum detection error probability, and expected detection error probability can be obtained.

The optimal detection threshold is expressed as follows:

$$
\tau^{*}= \begin{cases}{\left[\rho_{1}, \rho_{2}\right],} & \rho_{1}<\rho_{2}, \\ {\left[\rho_{2}, \rho_{1}\right],} & \rho_{1} \geq \rho_{2} .\end{cases}
$$
follows:

The minimum detection error probability is expressed as

$$
\xi^{*}= \begin{cases}0, & \rho_{1}<\rho_{2}, \\ 1-\frac{P_{A}\left|h_{A W}\right|^{2}}{P_{R_{j}}^{\max }\left|h_{R_{j} W}\right|^{2}}, & \rho_{1} \geq \rho_{2},\end{cases}
$$

where $\rho_{1} \triangleq P_{R_{j}}^{\max }\left|h_{R_{j} W}\right|^{2}+\sigma_{W}^{2}, \rho_{2} \triangleq P_{A}\left|h_{A W}\right|^{2}+\sigma_{W}^{2}, \rho_{3} \triangleq P_{A}$ $\left|h_{A W}\right|^{2}+P_{R_{j}}^{\max }\left|h_{R_{i} W}\right|^{2}+\sigma_{W}^{2}$.

The expected detection error probability is expressed as follows:

$$
\overline{\xi^{*}}(t)=-t^{2}+t \ln t+1
$$

where

$$
t \triangleq \frac{P_{A}\left|h_{A W}\right|^{2}}{P_{A}\left|h_{A W}\right|^{2}+P_{R_{j}}^{\max }\left|h_{R_{j} W}\right|^{2}} .
$$

It can be seen from [2] that the expected detection error rate $\overline{\xi^{*}}$ is a monotone increasing function of $P_{R_{j}}^{\max }$.

3.4. Covert Performance. In general, the constraint for covert transmission can be defined as $\xi^{*} \geq 1-\varepsilon$, where $\varepsilon$ is a predetermined value, and there is $\varepsilon \in[0,1]$. Therefore, the maximized effective covert rate can be expressed as follows:

$$
\begin{aligned}
& \max _{P_{Z}} \overline{R_{C}}, \\
& \text { s.t. } \overline{\xi^{*}} \geq 1-\varepsilon,
\end{aligned}
$$

where $\overline{R_{C}} \triangleq R(1-\delta)$.
3.4.1. The Transmission Outage Probability of Alice to Relay. Assuming that the transmission rate $R$ is known, according to formula (1), $\left|h_{A R_{i}}\right|^{2},\left|h_{R_{j} R_{j}}\right|^{2}$ and $P_{R_{j}}$ are random variables, which can still cause transmission interruption.

Since the wireless channel is subject to independent quasi-static Rayleigh fading and independent identically distributed, the cumulative distribution function $(C D F)$ of $\max _{1 \leq i \leq N_{R}}\left(\left|h_{A R_{i}}\right|^{2}\right)$ is $F_{1 \leq i \leq N_{R}}\left(\left|h_{A R_{i}}\right|^{2}\right)$
its PDF is

$$
f_{\max _{1 \leq i \leq N_{R}}\left(\left|h_{A R_{i}}\right|^{2}\right)}(x)=\sum_{m=0}^{N_{R}}\left(\begin{array}{c}
N_{R} \\
m
\end{array}\right)(-1)^{m}\left(e^{-x}\right)^{m} .
$$

Theorem 1. For the first stage, the transmission outage probability from Alice to Relay is derived as follows:

$$
\begin{aligned}
\delta_{1}= & 1+\frac{P_{A}}{P_{R_{j}}^{\max } \varphi\left(2^{2 R}-1\right)} \sum_{m=1}^{N_{R}}\left(\begin{array}{c}
N_{R} \\
m
\end{array}\right)(-1)^{m} \frac{1}{m}, \\
& \exp \left[-\frac{m\left(2^{2 R}-1\right) \sigma_{R}^{2}}{P_{A}}\right] \times \ln \left[1+m \varphi\left(2^{2 R}-1\right) \frac{P_{R_{j}}^{\max }}{P_{A}}\right] .
\end{aligned}
$$

Proof. see Appendix A.

Remark 1. The comments are as follows:

(1.1) In the first stage, the maximum power $P_{R_{j}}^{\max }$ of transmitting AN of a single antenna randomly selected by Relay can have a direct influence on the transmission outage probability $\delta_{1}$. The larger $P_{R_{j}}^{\max }$ is, the greater $\delta_{1}$ will be.

(1.2) In the first stage, the transmission outage probability $\delta_{1}$ is a monotonically increasing function of channel noise $\sigma_{R}^{2}$ and transmission rate $R$, that is, the larger $\sigma_{R}^{2}$ and $R$ are, the larger $\delta_{1}$ will be.

(1.3) The configuration of antenna number $N_{R}$ has a direct influence on the transmission outage probability $\delta_{1}$ of the first stage. The larger $N_{R}$ is, the smaller $\delta_{1}$ is.

(1.4) In the first stage, the transmission outage probability $\delta_{1}$ is a monotonically decreasing function of Alice's covert message sending power $P_{A}$, that is, the larger $P_{A}$ is, the smaller $\delta_{1}$ is. And the transmission outage probability $\delta_{1}$ is a monotonically increasing function of the self-interference elimination coefficient $\phi$, that is, the larger $\phi$ is, the larger $\delta_{1}$ is.

\subsubsection{The Optimal AN}

Theorem 2. Under any given fixed covert information power $P_{A}$ sent by Alice, predetermined $\varepsilon$ and transmission rate $R$, the optimal AN power sent by Relay is given by 


$$
P_{R_{j}}^{\max ^{*}}=\frac{\left(1-t_{\varepsilon}\right) P_{A}\left|h_{A W}\right|^{2}}{\left|h_{R_{j} W}\right|^{2} t_{\varepsilon}}
$$

where $t_{\varepsilon}$ is the solution of $\overline{\xi^{*}}(t)=1-\varepsilon$.

\subsubsection{The Maximized Effective Covert Rate}

Theorem 3. The optimal effective convert rate is derived as follows:

Proof. see Appendix B.

$$
\overline{R_{C_{1}}^{*}}=-R\left|h_{R_{j} W}\right|^{2} t_{\varepsilon} \sum_{m=1}^{N_{R}}\left(\begin{array}{c}
N_{R} \\
m
\end{array}\right)(-1)^{m} \times \exp \left[-\frac{m\left(2^{2 R}-1\right) \sigma_{R}^{2}}{P_{A}}\right] \frac{\ln \left[1+m \varphi\left(2^{2 R}-1\right)\left(1-t_{\varepsilon}\right)\left|h_{A W}\right|^{2} / t_{\varepsilon}\left|h_{R_{j} W}\right|^{2}\right]}{m \varphi\left(2^{2 R}-1\right)\left(1-t_{\varepsilon}\right)\left|h_{A W}\right|^{2}} .
$$

Proof. see Appendix C.

Remark 2. The comments are as follows:

(2.1) The larger the predetermined convert constraint $\varepsilon$ in the first stage, the greater the maximum effective convert rate of the first stage.

Due to $\overline{R_{C}} \triangleq R(1-\delta)$, then there is $\delta_{1}^{*}$, so there will be $\overline{R_{C_{1}}^{*}} \triangleq R\left(1-\delta_{1}^{*}\right)$, and $\delta_{1}^{*}$ also has the property of $\delta_{1}$, so we can obtain Remarks 2.2-2.4.

(2.2) In the first stage, the maximum effective covert rate $\overline{R_{C_{1}}^{*}}$ increases with the increase of the antenna number $N_{R}$ and the power $P_{A}$ of covert messages sent by Alice.

(2.3) In the first stage, the maximum effective covert rate $\overline{R_{C}^{*}}$ is the monotonic decreasing function of the channel noise $\sigma_{R}^{2}$ of Relay and its self-interference elimination coefficient $\phi$, that is, the larger $\sigma_{R}^{2}$ and $\phi$ are, the smaller $\overline{R_{C_{1}}^{*}}$ is.

(2.4) In the first stage, the maximum effective covert rate $\overline{R_{C}^{*}}$ is the monotonic decreasing function of the channel coefficient $\left|h_{A W}\right|^{2}$, that is, the larger $\left|h_{A W}\right|^{2}$ is, the smaller $R_{C_{1}}^{*}$ is. And the maximum effective covert rate $\overline{R_{C_{1}}^{*}}$ is the monotonic increasing function of the $\left|h_{R_{j} W}\right|^{2}$, that is, the larger $\left|h_{R_{j} W}\right|^{2}$ is, the larger $\overline{R_{C_{1}}^{*}}$ is.

Corollary 1. In the first stage, if the power $P_{A}$ of Alice sending covert messages to Relay increases, the maximum effective covert rate $\overline{R_{C_{1}}^{*}}$ tends to a fixed value:

$$
\begin{aligned}
\overline{R_{C_{1}}^{*}} & =R\left|h_{R_{j} W}\right|^{2} t_{\varepsilon} \sum_{m=1}^{N_{R}}\left(\begin{array}{c}
N_{R} \\
m
\end{array}\right)(-1)^{m}, \\
& \frac{\ln \left[1+m \varphi\left(2^{2 R}-1\right)\left(1-t_{\varepsilon}\right)\left|h_{A W}\right|^{2} / t_{\varepsilon}\left|h_{R_{j} W}\right|^{2}\right]}{m \varphi\left(2^{2 R}-1\right)\left(1-t_{\varepsilon}\right)\left|h_{A W}\right|^{2}} .
\end{aligned}
$$

Proof. when $P_{A}$ approaches $\infty$, then $\exp \left\{-\left[m\left(2^{2 R}\right.\right.\right.$ - 1) $\left.\left.\sigma_{R}^{2}\right] / P_{A}\right\}$ approaches 1 , so the maximum effective convert rate approaches the result in formula (18) in the first stage.

\section{The Second Stage: Relay Forwards Covert Messages to Bob}

Relay forwards Alice's information to Bob and sends zeroforcing AN signal to Willie. Relay selects the best antenna according to the CSI between it and Bob and decodes and forwards the convert messages sent by Alice to Bob, and the remaining $N_{R}-1$ antennas are all used to send zero-forcing AN to Willie.

Relay works in the decoding and forwarding mode. In the second stage, Relay decodes and encodes the received signal and forwards it to Bob, whose transmitted signal is $X_{R}[i]$. The $P_{A}$ is fixed, and Relay's forwarding mode is decoded forwarding, so it is assumed that Alice's covert messages power is also fixed by Relay forwarding; both Bob and Willie know this.

4.1. Reception at Bob. The instantaneous SINR at Bob is given by

$$
\gamma_{B o b}=\frac{P_{R_{k}} \max _{1 \leq k \leq N_{R}}\left(\left|h_{R_{k} B}\right|^{2}\right)}{\sigma_{B}^{2}}
$$

where $h_{R_{k} B}$ is the channel coefficient between the $k$-th antenna selected by Relay and Bob; here, $k=1,2, \ldots, N_{R}, P_{R_{k}}$ is the fixed power of Relay forwarding Alice covert messages; $\sigma_{B}^{2}$ is the channel variance of Relay.

In the second stage, when Relay sends covert messages, the signal received by Bob can be expressed as follows:

$$
y_{B o b}[i]=\sqrt{P_{R_{k}}} \max _{1 \leq k \leq N_{R}}\left(\left|h_{R_{k} B}\right|\right) x_{R}[i]+n_{B}[i],
$$

where $x_{R}[i]$ is the covert signal forwarded by Relay, satisfying $E\left[x_{R}[i] x_{R}^{\dagger}[i]\right]=1 ; i=1,2, \ldots, n$ represents the symbol index, $n$ is the total number of channels used in each slot, and $n_{B}[i]$ is the AWGN at Bob with $\sigma_{B}^{2}$ as its variance, i.e., $n_{B}[i] \sim C N\left(0, \sigma_{B}^{2}\right)$. 
4.2. Detection Metrics at Willie. In order to cause the uncertainty of Willie's detection power, Relay uses the remaining $N_{R}-1$ antennas for zero-forcing beamforming, which interferes with Willie's transmission without affecting Bob's reception. The optimal weighted vector $\mathbf{W}_{Z F}$ is the solution of the following optimization problem:

$$
\begin{aligned}
& \max _{\mathbf{W}_{Z F}}\left|h_{R W}^{\dagger} \mathbf{W}_{Z F}\right|, \\
& \text { s.t. }\left|h_{R B}^{\dagger} \mathbf{W}_{Z F}\right|=0 \quad\left\|\mathbf{W}_{Z F}\right\|_{F}=1,
\end{aligned}
$$

where $\dagger$ is the conjugate transpose operator, $\|\bullet\|_{F}$ denotes the Frobenius norm, and $h_{R W}$ or $h_{R B}$ represents the $n$-dimensional channel vectors between Relay and Willie or Bob, respectively. According to the theorems in $[40,41]$, the solution of the optimization problem in formula (21), i.e., the precoding vector $\mathbf{W}_{Z F}$, can be described as follows:

$$
\mathbf{W}_{Z F}=\frac{T^{\perp} h_{R W}}{\left\|T^{\perp} h_{R W}\right\|},
$$

where $T^{\perp}=\left(I-h_{R B}\left(h_{R B}^{\dagger} h_{R B}\right)^{-1} h_{R B}^{\dagger}\right)$ is the projection idempotent matrix with rank $N_{R}-2$.

Let us define $Z_{1} \triangleq P_{Z}\left|h_{R W}^{\dagger} W_{Z F}\right|^{2} / \sigma_{W}^{2}$ and $Z \triangleq P_{Z}\left|h_{R W}^{\dagger} W_{Z F}\right|^{2}$. According to equations (11) and (12) in [40], we have

$$
\begin{gathered}
f_{Z_{1}}(Z)=\frac{Z^{N_{R}-3} \exp \left(-\sigma_{w}^{2} Z / P_{Z}\right)}{\left(N_{R}-3\right) !\left(P_{Z} / \sigma_{w}^{2}\right)^{N_{R}-2}}, \quad N_{R} \geq 3, Z \geq 0, \\
F_{Z_{1}}(Z)=1-\exp \left(-\frac{Z \sigma_{W}^{2}}{P_{Z}}\right) \sum_{l=0}^{N_{R}-3} \frac{1}{l !}\left(\frac{Z \sigma_{W}^{2}}{P_{Z}}\right)^{l}, \quad Z \geq 0 .
\end{gathered}
$$

The SINR at Willie is given by

$$
\gamma_{\text {willie } 2}=\frac{P_{R_{k}}\left|h_{R_{k} W}\right|^{2}}{P_{Z}\left|h_{R W}^{\dagger} W_{Z F}\right|+\sigma_{W}^{2}},
$$

where $h_{R_{k} W}$ is the channel coefficient between the $k$-th antenna that Relay chooses to forward the covert messages and Bob and $P_{Z}$ is the AN power of ZFB sent by Relay. In a communication slot of the second stage, Willie has to decide whether Relay has forwarded covert messages to Bob [42]. Therefore, Willie is faced with a binary hypothesis testing problem again, where the zero hypothesis $M_{0}$ means Relay has not forwarded covert messages, and the alternative hypothesis $M_{1}$ means Relay has forwarded covert messages to Bob. Based on these assumptions, Willie receives signals is given by

$$
y_{\text {willie } 2}[i]= \begin{cases}\sqrt{P_{Z}}\left|h_{R W}^{\dagger} W_{Z F}\right| V_{R_{2}}[i]+n_{W}[i], & M 0, \\ \sqrt{P_{R_{k}}}\left|h_{R_{k} W}\right| x_{R}[i]+\sqrt{P_{Z}}\left|h_{R W}^{\dagger} W_{Z F}\right| V_{R_{2}}[i]+n_{W}[i], & M 1 .\end{cases}
$$

Willie does not know the value of $P_{Z}$ in the time slot, but the value of $P_{R_{K}}$ is known. In the second stage, Willie attempts to detect whether $y_{\text {willie } 2}$ is $M_{0}$ or $M_{1}$. Through the application of Neyman-Perason criterion and likelihood ratio test, the optimal decision rule for Willie to minimize his detection error is as follows:

$$
T_{W 2} \underset{U_{1}}{\stackrel{U_{0}}{>}} \tau
$$

where $T_{W 2}=1 / n \sum_{i=1}^{n} y_{W i l l i e 2}[i]$ is the average power received at Willie in the slot, $\tau$ is a predefined Willie's detection threshold, and $U_{0}$ and $U_{1}$ are binary decisions that infer whether Relay forwards covert messages, respectively. In this paper, we consider an infinite number of channel uses, that is, $n \longrightarrow \infty$. Therefore, we have

$$
T_{W 2}= \begin{cases}P_{Z}\left|h_{R W}^{\dagger} W_{Z F}\right|^{2}+\sigma_{W}^{2}, & U 0, \\ P_{R_{k}}\left|h_{R_{k} W}\right|^{2}+P_{Z}\left|h_{R W}^{\dagger} W_{Z F}\right|^{2}+\sigma_{W}^{2}, & U 1 .\end{cases}
$$

At the end of the communication slot, Willie has to make a decision. The false alarm rate is defined as the probability of Willie making $U_{1}$ decision under condition $M_{0}$, which is expressed by $\alpha_{2}=P\left(U_{1} \mid M_{0}\right)$. Similarly, the miss detection rate is defined as the probability of Willie making $U_{0}$ decision under condition $M_{1}$, which is expressed by $\beta_{2}=P\left(U_{0} \mid M_{1}\right)$. Assuming that the prior probabilities of $M_{0}$ and $M_{1}$ are equal, Willie's detection performance can be judged by detecting error rate, which can be defined as follows:

$$
\xi_{2} \triangleq \alpha_{2}+\beta_{2}
$$

\subsection{Detection Performance at Willie}

\subsubsection{False Alarm Rate and Miss Detection Rate}

Theorem 4. In the second stage, Willie's false alarm rate is derived as follows: 


$$
\alpha_{2}= \begin{cases}1, & \tau \leq \sigma_{W}^{2}, \\ x \operatorname{Ei}(-x)+\exp (-x), & \tau>\sigma_{W}^{2} N_{R}=3, \\ \exp (-x), & \tau>\sigma_{W}^{2} N_{R}=4, \\ \left\{1+\sum_{l=2}^{N_{R}-3}\left[\frac{1}{l(l-1)} \sum_{k=0}^{l-2} \frac{x^{k+1}}{k !}\right]\right\} \exp (-x), & \tau>\sigma_{W}^{2} N_{R} \geq 5,\end{cases}
$$

and the miss detection rate is formula (31) where $x=\left(\tau-\sigma_{w}^{2}\right.$ $\left./\left(N_{R}-1\right) P_{R_{j}}^{\max }\right), y=\left(\tau-\sigma_{w}^{2}-P_{R_{k}}\left|h_{R_{k} W}\right|^{2} /\left(N_{R}-1\right) P_{R_{j}}^{\max }\right)$.

The $P_{R_{i}}^{\max }$ is the maximum AN power transmitted by Relay's single antenna, which has been introduced in Section 3.1.

$$
\beta_{2}= \begin{cases}0, & \tau \leq \sigma_{W}^{2}+P_{R_{k}}\left|h_{R_{k} W}\right|^{2}, \\ 1-[y \operatorname{Ei}(-y)+\exp (-y)], & \tau>\sigma_{W}^{2}+P_{R_{k}}\left|h_{R_{k} W}\right|^{2} N_{R}=3, \\ 1-\exp (-y), & \tau>\sigma_{W}^{2}+P_{R_{k}}\left|h_{R_{k} W}\right|^{2} N_{R}=4, \\ 1-\left\{1+\sum_{l=2}^{N_{R}-3}\left[\frac{1}{l(l-1)} \sum_{k=0}^{l-2} \frac{y^{k+1}}{k !}\right]\right\} \exp (-y), & \tau>\sigma_{W}^{2}+P_{R_{k}}\left|h_{R_{k} W}\right|^{2} N_{R} \geq 5 .\end{cases}
$$

Proof. see Appendix D.

\subsubsection{Optimal Detection Threshold and Minimum Detection Error Rate}

Theorem 5. According to Willie's hypothesis, the optimal detection threshold can be expressed as follows:

$$
\tau^{*}=\sigma_{W}^{2}+P_{R_{K} W}\left|h_{R_{K} W}\right|^{2} .
$$

The corresponding minimum detection error rate is derived as follows:

$$
\xi_{2}^{*}= \begin{cases}x^{*} E i\left(-x^{*}\right)+\exp \left(-x^{*}\right), & N_{R}=3, \\ \exp \left(-x^{*}\right), & N_{R}=4, \\ \left\{1+\sum_{l=2}^{N_{R}-3}\left[\frac{1}{l(l-1)} \sum_{k=0}^{l-2} \frac{x^{* k+1}}{k !}\right]\right\} \exp \left(-x^{*}\right), & N_{R} \geq 5,\end{cases}
$$

where

$$
x^{*}=\frac{P_{R_{k}}\left|h_{R_{k} W}\right|^{2}}{\left(N_{R}-1\right) P_{R_{j}}^{\max } \lambda_{R W}} .
$$

Proof. see Appendix E.

Remark 3. The comments are as follows:

(3.1) In the second stage, the minimum detection error rate $\xi_{2}^{*}$ is a monotonic increasing function of the maximum AN power $P_{R_{j}}^{\max }$ of an antenna in Relay using ZFB method, that is, the larger $P_{R_{j}}^{\max }$ is, the larger $\xi_{2}^{*}$ is. The minimum detection error rate $\xi_{2}^{*}$ is also monotonically increasing with respect to the number of antennas $N_{R}$, that is, the larger $N_{R}$ is, the larger $\xi_{2}^{*}$ is.

(3.2) In the second stage, the minimum detection error rate $\xi_{2}^{*}$ is a monotonic decreasing function of Relay forwarding covert message power $P_{R_{k}}$, that is, the larger $P_{R_{k}}$ is, the smaller $\xi_{2}^{*}$ is.

4.4. Covert Performance. In the second stage, the maximized effective covert rate can be expressed as follows:

$$
\begin{array}{ll}
\underset{P_{R_{k}}}{\arg \max } & \overline{R_{C}}, \\
\text { s.t. } & \xi_{2}^{*} \geq 1-\varepsilon .
\end{array}
$$

4.4.1. The Transmission Outage Probability of Relay to Bob. Assuming that the transmission rate $R$ is known, according to formula (19), $\left|h_{R_{k} B}\right|^{2}$ and $P_{R_{K}}$ are random variables, which can still cause transmission interruption.

Since the wireless channel is subject to independent quasi-static Rayleigh fading and independent identically distributed, the $(C D F)$ of $\max _{1 \leq i \leq N_{R}}\left(\left|h_{R_{k} B}\right|^{2}\right)$ is $F_{1 \leq k \leq N_{R}}\left(\left|h_{R_{k} B}\right|^{2}\right)$
$(x)=\left(1-e^{-x}\right)^{N_{R}}$; then its PDF is

$$
f \max _{1 \leq k \leq N_{R}}\left(\left|h_{R_{k} B}\right|^{2}\right)(x)=\sum_{m=0}^{N_{R}}\left(\begin{array}{c}
N_{R} \\
m
\end{array}\right)(-1)^{m}\left(\mathrm{e}^{-x}\right)^{m} .
$$


Theorem 6. For the second stage, the transmission outage probability from Relay to Bob is derived as follows:

$$
\delta=\left[1-\exp \left(-\frac{\left(2^{2 R}-1\right) \sigma_{B}^{2}}{P_{R_{k}}}\right)\right]^{N_{R}} .
$$

Proof. see Appendix F.

Remark 4. The comments are as follows:

(4.1) In the second stage, the larger the power $P_{R_{b}}$ of Relay forwarding Alice covert messages is, the smaller the transmission outage probability $\delta_{2}$ is

(4.2) In the second stage, the transmission outage probability $\delta_{2}$ is a monotonically increasing function of $\sigma_{B}^{2}$ and $R$, that is, the larger $\sigma_{B}^{2}$ and $R$ are, the greater $\delta_{2}$ is

(4.3) In the second stage, the transmission outage probability $\delta_{2}$ decreases with the increase of the number of antennas $N_{R}$, that is, the larger $N_{R}$ is, the smaller $\delta_{2}$ is

\subsubsection{Optimal Transmission Power of Relay Forwarding Covert Messages}

Theorem 7. Under any given $\varepsilon$ and transmission rate $R$, the optimal transmission power of Relay forwarding Alice's covert message is expressed as follows:

$$
P_{R_{k}}^{*}= \begin{cases}\frac{\left(N_{R}-1\right) P_{R_{j}}^{\max } t_{\varepsilon_{1}}}{\left|h_{R_{k} W}\right|^{2}}, & N_{R}=3, \\ \frac{\left(N_{R}-1\right) P_{R_{j}}^{\max }}{\left|h_{R_{k} W}\right|^{2}} \ln \left(\frac{1}{1-\varepsilon}\right), & N_{R}=4, \\ \frac{\left(N_{R}-1\right) P_{R_{j}}^{\max } t_{\varepsilon_{2}}}{\left|h_{R_{k} W}\right|^{2}}, & N_{R} \geq 5,\end{cases}
$$

where $t_{\varepsilon_{1}}$ is the solution of $x E i(-x)+\exp (-x)=1-\varepsilon$ and $t_{\varepsilon_{2}}$ is the solution of $\left\{1+\sum_{l=2}^{N_{R}-3}\left[1 / l(l-1) \sum_{k=0}^{l-2} x^{k+1} / k !\right]\right\} \exp (-x)=1-\varepsilon$.

Proof: see Appendix G.

\subsubsection{Optimal Effective Convert Rate}

Theorem 8. In the second stage, the optimal effective convert rate is derived as follows:

$$
{\overline{R_{C_{2}}}}^{*}=R-R\left\{1-\exp \left[-\frac{\left(2^{2 R}-1\right) \sigma_{B}^{2}}{P_{R_{k}}^{*}}\right]\right\}^{N_{R}} .
$$

Proof. see Appendix H.

Remark 5. The comments are as follows:

(5.1) In the second stage, the optimal effective convert rate $\overline{R_{C}^{*}}$ is a monotonic increasing function of the AN power $P_{R_{j}}^{\max }$, that is, the larger $P_{R_{j}}^{\max }$ is, the larger $\overline{R_{C_{2}}^{*}}$ is (5.2) In the second stage, the optimal effective convert rate $\overline{R_{C_{2}}^{*}}$ increases with the increase of antenna number $N_{R}$ and $\varepsilon$, that is, the larger $N_{R}$ and $\varepsilon$, the greater $\overline{R_{C_{2}}^{*}}$ (5.3) In the second stage, the optimal effective convert rate $\overline{R_{C_{2}}^{*}}$ increases with the increase of the convert message power $P_{\mathrm{R}_{k}}^{*}$ forwarded by Relay, and it decreases with the increase of channel noise $\sigma_{B}^{2}$ and channel coefficient $\left|h_{R_{k} W}\right|^{2}$

Corollary 2. In the second stage, if the optimal power of Relay forwarding Alice's covert messages is increased, the maximum effective covert rate approaches a fixed value $R$.

$$
\lim _{R_{k}} \longrightarrow \infty \overline{R_{C_{2}}^{*}}=R
$$

Proof. according to formula (39), when $P_{\mathrm{R}_{k}}^{*}$ tends to $\infty$, the $\exp \left\{-\left[\left(2^{2 R}-1\right) \sigma_{B}^{2}\right] / P_{R_{k}}^{*}\right\}$ tends to 1 ; then $\{1-\exp$ $\left.\left[-\left(2^{2 R}-1\right) \sigma_{B}^{2} / P_{R_{k}}^{*}\right]\right\}^{N_{R}} \longrightarrow 0$, so $\overline{R_{C_{2}}^{*}} \longrightarrow R$.

\section{Total Performance}

\subsection{Transmission Outage Probability}

Theorem 9. The total transmission outage probability is formula (41).

$$
\begin{aligned}
\delta= & 1+\frac{P_{A}}{P_{R_{j}}^{\max } \varphi\left(2^{2 R}-1\right)} \sum_{m=1}^{N_{R}}\left\{\left(\begin{array}{c}
N_{R} \\
m
\end{array}\right)(-1)^{m} \frac{1}{m} \exp \left[-\frac{m\left(2^{2 R}-1\right) \sigma_{R}^{2}}{P_{A}}\right] \ln \left[1+m \varphi\left(2^{2 R}-1\right) \frac{P_{R_{j}}^{\max }}{P_{A}}\right]\right\} \\
& \times\left\{1-\left[1-\exp \left(-\frac{\left(2^{2 R}-1\right) \sigma_{B}^{2}}{P_{R_{k}}}\right)\right]^{N_{R}}\right\} .
\end{aligned}
$$


Proof. see Appendix I.

Theorem 10. The $\sigma_{R}^{2}=\sigma_{B}^{2}, P_{A}=P_{R_{k}}^{*}$ is assumed; then the overall optimal effective covert rate is given by

\subsection{Maximum Effective Covert Rate}

$$
\begin{aligned}
\overline{R_{C}^{*}}= & \overline{R_{C_{1}}^{*}}=-R\left|h_{R_{j} W}\right|^{2} t_{\varepsilon} \sum_{m=1}^{N_{R}}\left(\begin{array}{c}
N_{R} \\
m
\end{array}\right)(-1)^{m}, \\
& \exp \left[-\frac{m\left(2^{2 R}-1\right) \sigma_{R}^{2}}{P_{A}}\right] \frac{\ln \left[1+m \varphi\left(2^{2 R}-1\right)\left(1-t_{\varepsilon}\right)\left|h_{A W}\right|^{2} / t_{\varepsilon}\left|h_{R_{j} W}\right|^{2}\right]}{m \varphi\left(2^{2 R}-1\right)\left(1-t_{\varepsilon}\right)\left|h_{A W}\right|^{2}} .
\end{aligned}
$$

Proof. see Appendix J.

\section{Numerical Result}

6.1. The First Stage. In this section, we present some detailed numerical results to illustrate the influence of system parameters on the detection and covert performance of the system. By observing the results in these figures, it can be found that the analysis results are completely consistent with the derivation results, which verifies the correctness of the theoretical analysis. If there are no special cases, we will set some general parameter values. Due to the channel does not change in a time slot, that is, it remains a constant, the value of the channel coefficient can be set to $0 \mathrm{~dB}$. At the same time, the value of channel noise is set to $0 \mathrm{~dB}$. The transmitting power of a single antenna (including nodes with a single antenna) is set to $10 \mathrm{~dB}$. We define the predetermined covert communication condition value as 0.05 and determine the transmission rate as 1 or 1.5. Specific simulation data can be referred to in the instructions below each figure.

6.1.1. Transmission Outage Probability. Figures 2-4 illustrate the relationship between the transmission outage probability $\delta_{1}$ and the maximum power $P_{R_{j}}^{\max }$ of a single antenna randomly selected by Relay based on different parameter values. It can be observed that the larger $P_{R_{j}}^{\max }$ is, the greater $\delta_{1}$ is. This is because Relay will also generate selfinterference while sending interference to Willie. Such selfinterference will affect the communication between Alice and the $i$-th antenna of Relay, and the transmission is more likely to be interrupted. This is consistent with Remark 1.1. In Figure 2, it can be observed that the transmission outage probability $\delta_{1}$ is a monotonic increasing function with respect to $\sigma_{R}^{2}$. This is because the larger the channel noise of Relay is, the smaller the SINR of the channel is, and the easier the transmission is interrupted. It can also be seen from Figure 2 that $\delta_{1}$ is a monotonic increasing function of $R$, that is, the higher the transmission rate is, the easier the transmission is interrupted. These observations are consistent with Remark 1.2. In Figure 3, with the increase of the number of antennas, the transmission outage probability $\delta_{1}$ is smaller. This is because the more the number of antennas is configured, the more security gain can be obtained, so that the more secure the transmission is, the less likely the transmission is interrupted. This confirms the correctness of
Remark 1.3. In Figure 4, we can see that $\delta_{1}$ is a monotonic increasing function of $\phi$, that is, the larger the interference cancellation coefficient is, the easier the transmission is interrupted. This is because the self-interference coefficient determines the degree of self-interference. The greater the self-interference is, the less secure the transmission from Alice to Relay is. At the same time, it can be observed from Figure 4 that $\delta_{1}$ is a monotonic decreasing function of $P_{A}$. The higher the power of hidden message transmission, the more favorable it is for Relay's reception, and the lower the transmission outage probability. This is consistent with Remark 1.4. In addition, since the expected detection error rate is cited in [2], it can be seen from Figure 3 of [2]: (1) the expected detection error rate $\overline{\xi^{*}}$ is a monotone increasing function of $P_{R_{j}}^{\max }$ and (2) the expected detection error rate $\bar{\xi}^{*}$ is a monotonic decreasing function of $P_{A}$.

6.1.2. Maximum Effective Convert Rate. Figures 5-7 shows the probability simulation curves of the maximum effective convert rate $\overline{R_{C_{1}}^{*}}$ under different power $P_{A}$ that Alice sends covert messages. It can be observed that $R_{C}^{*}$ increases with the increase of $P_{A}$. This is because the larger the transmission power of the covert message, the larger the SINR of the antenna used to receive the covert message at Relay, and the smaller the transmission outage probability in the first stage, so that $\overline{R_{C_{1}}^{*}}$ will be larger. It can be observed from Figure 5 that the larger $\varepsilon$ is, the larger the maximum effective covert rate is. This is because the larger $\varepsilon$ is and the smaller $1-\varepsilon$ is, the lower Willie's minimum detection error rate is required, and the constraint condition of the maximum effective covert rate is more relaxed. Therefore, the maximum effective covert rate $\overline{R_{C_{1}}^{*}}$ is a monotonic increasing function of $\varepsilon$, which is consistent with the content of Remark 2.1. It can also be observed from Figure 5 that with the increase of the antenna's number $N_{R}$, the maximum effective covert rate increases. The reason is that the more the number of antennas, the smaller the transmission outage probability, but the larger the $\overline{R_{C_{1}}^{*}}$. This is consistent with Remark 2.2. It can be seen from Figure 6 that the maximum effective covert rate $\overline{R_{C_{1}}^{*}}$ is a monotonic decreasing function of $\sigma_{R}^{2}$ and $\phi$. The larger the channel noise of Relay, the smaller the SINR of Relay, the easier the transmission is interrupted, and the smaller the maximum effective covert rate will be. The larger the self-interference cancellation coefficient $\phi$, the greater the self-interference to Relay, and the greater the influence 


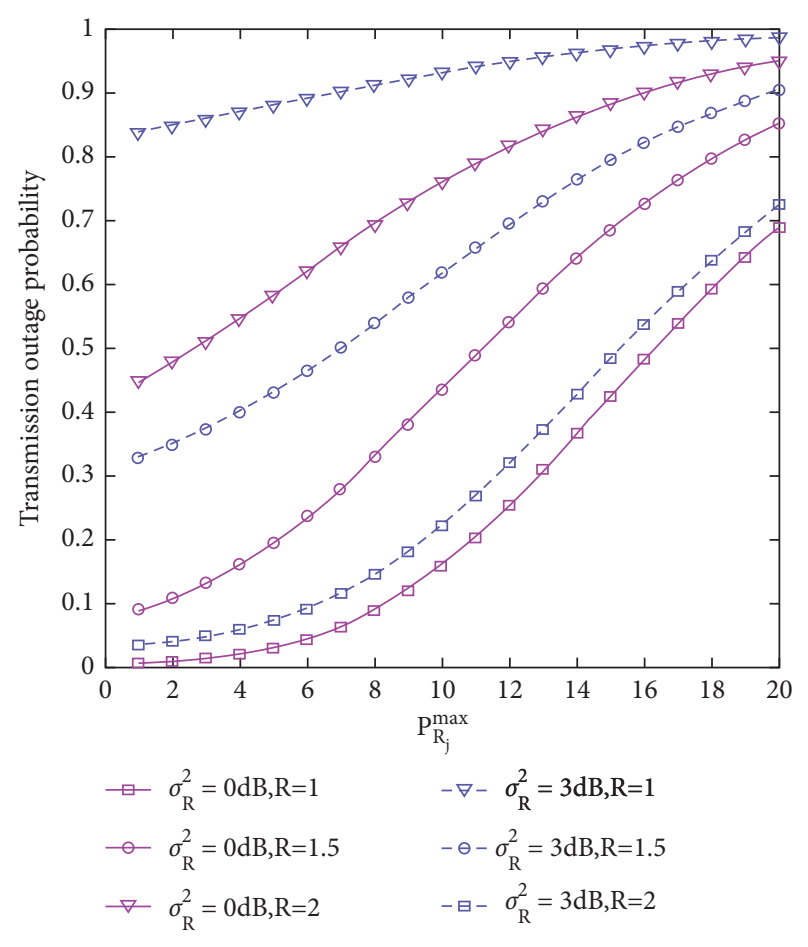

FIgURE 2: Transmission outage probability versus $P_{R_{j}}^{\max }$ with different value of $\sigma_{R}^{2}$ and $R$, where $P_{A}=10 d B, N_{R}=5$, and $\phi=0.5$.

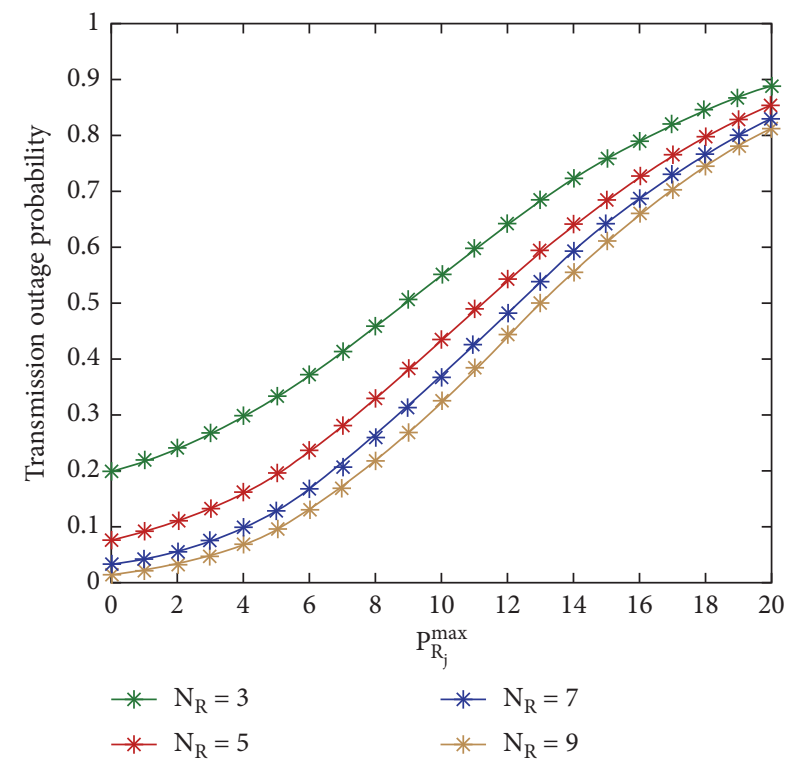

Figure 3: Transmission outage probability versus $P_{R_{i}}^{\max }$ with different value of $N_{R}$, where $P_{A}=10 \mathrm{~dB}, \sigma_{R}^{2}=0 \mathrm{~dB}, R=1.5$, and $\phi=0.5$.

on the covert transmission from Alice to Relay, so that the maximum effective covert rate $\overline{R_{C}^{*}}$ is smaller. This proves the correctness of inference Remark 2.3. Figure 7 illustrates the relationship between $\left|h_{A W}\right|^{2}$ and $\left|h_{R_{j} W}\right|^{2}$ and the maximum

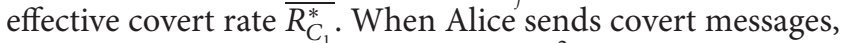
the larger the channel coefficient $\left|h_{A W}\right|^{2}$ between Alice and Willie is, the smaller the maximum effective covert rate $\overline{R_{C_{1}}^{*}}$ is. Because the larger the channel gain is, the more favorable

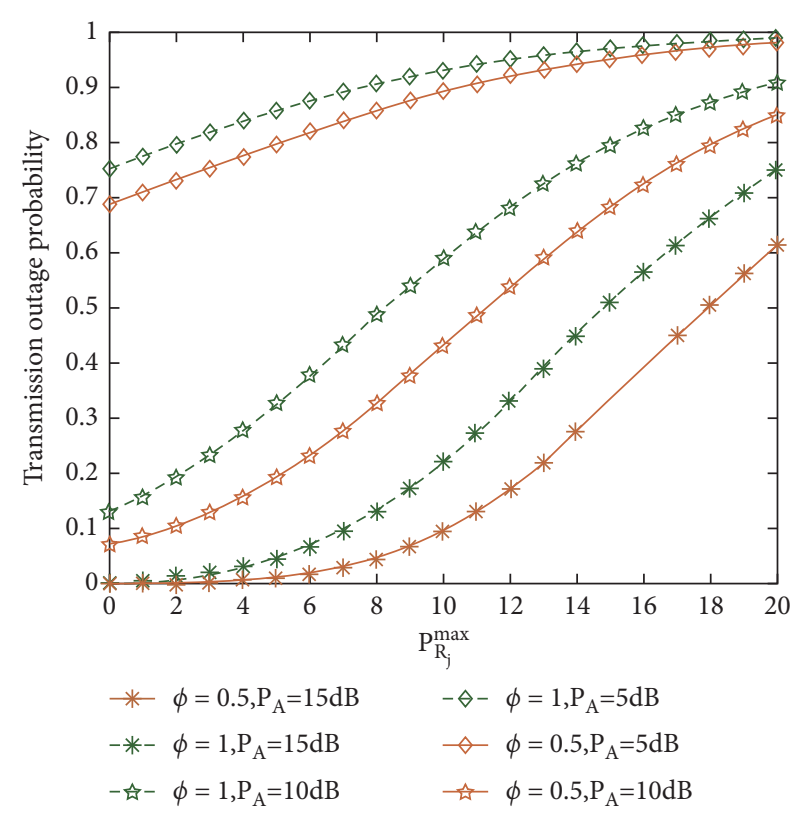

FIgURE 4: Transmission outage probability versus $P_{R_{j}}^{\max }$ with different value of $P_{A}$ and $\phi$, where $N_{R}=5, \sigma_{R}^{2}=0 d B$, and $R=1.5$.

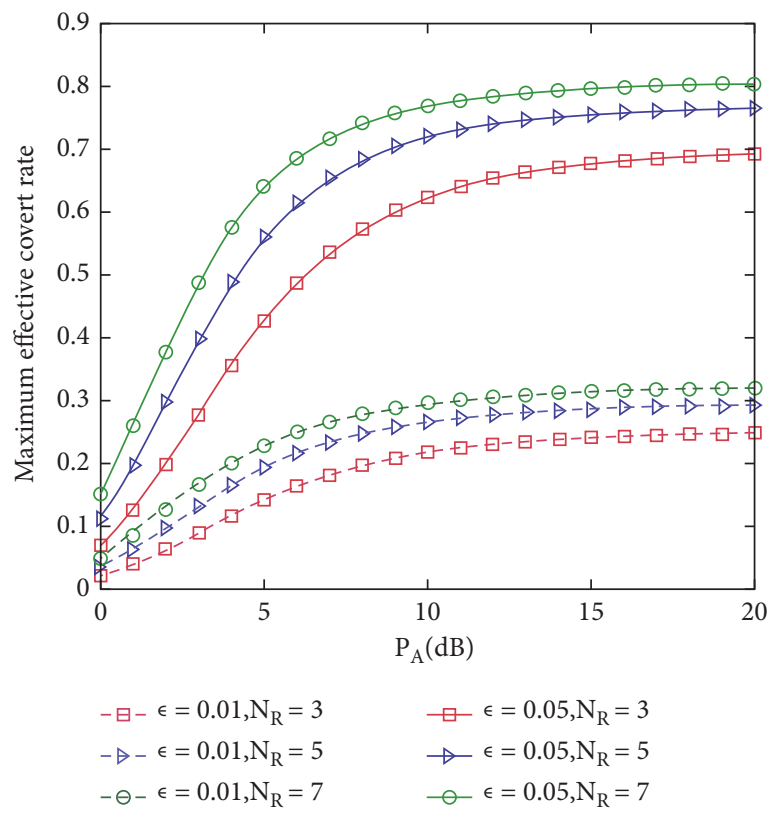

FIgURE 5: Maximum effective covert rate versus $P_{A}$ with different value of $\varepsilon$ and $N_{R}$, where $N_{R}=5, \sigma_{R}^{2}=0 d B, R=1,\left|h_{A W}\right|^{2}=1$, $\left|h_{R_{j} W}\right|^{2}=1$, and $\phi=0.01$.

Willie's detection is, the smaller the maximum effective covert rate is. And it can be observed that the larger the channel coefficient $\left|h_{R_{i} W}\right|^{2}$ between Relay randomly selected single antenna and Willie, the greater the maximum effective covert rate. The reason is that the more the channel gain of transmit interference is, the more difficult Willie is to detect, and the easier Willie is to perform covertness. This is consistent with Remark 2.4. In addition, a common point can be seen from Figures 5-7, that is, when increased to a certain value, the maximum effective covert rate of the first 


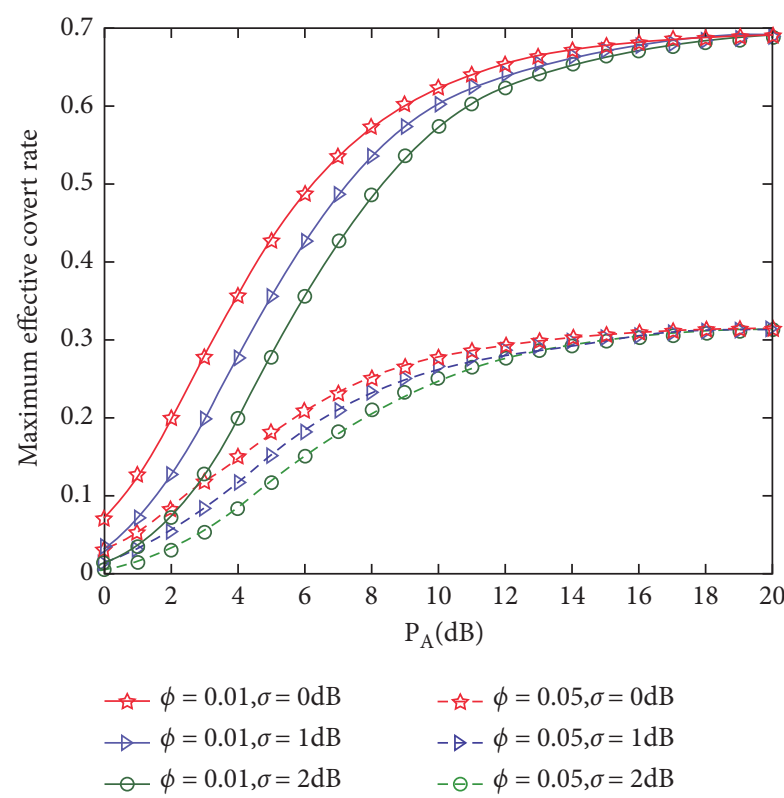

Figure 6: Maximum effective covert rate versus $P_{A}$ with different value of $\sigma_{R}^{2}$ and $\phi$, where $R=1,\left|h_{A W}\right|^{2}=1,\left|h_{R_{j} W}\right|^{2}=1, \varepsilon=0.05$, and $N_{R}=3$.

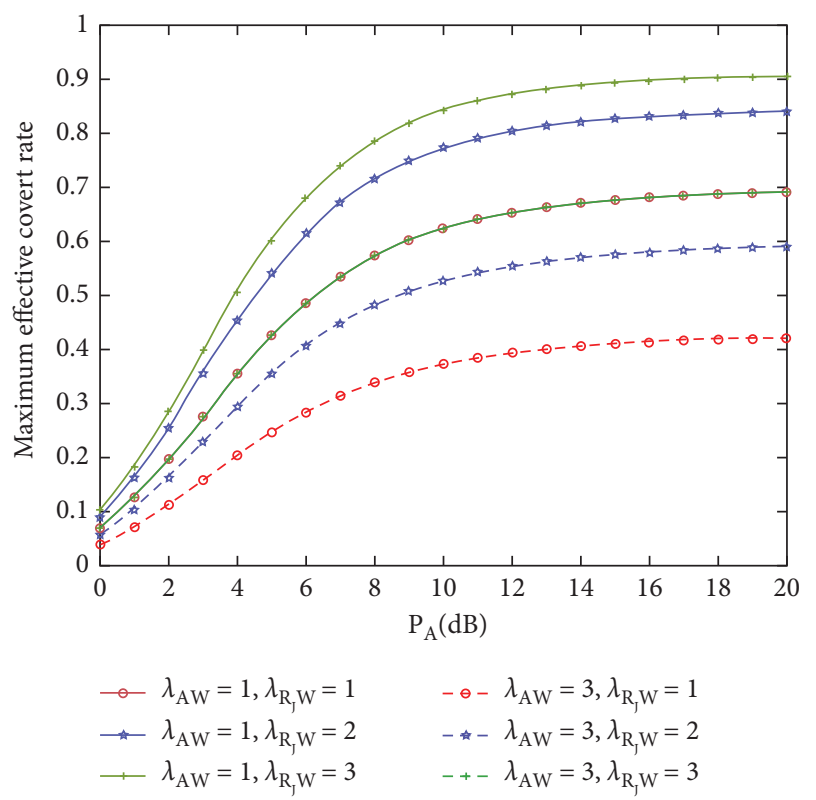

Figure 7: Maximum effective covert rate versus $P_{A}$ with different value of $\left|h_{A W}\right|^{2}$ and $\left|h_{R_{j} W}\right|^{2}$, where $R=1, \sigma_{R}^{2}=0 d B, \phi=0.01$, $\varepsilon=0.05$, and $N_{R}=3$.

stage tends to be a fixed value, which confirms the correctness of Corollary 1.

\subsection{The Second Stage}

6.2.1. Detection Error Probability. Figure 8 shows the relationship between the false alarm rate $\alpha_{2}$, missed detection rate $\beta_{2}$, detection error probability $\xi_{2}$, and the threshold $\tau$. It can be observed that the smaller the false alarm rate, the larger the missed detection rate, the smaller the missed

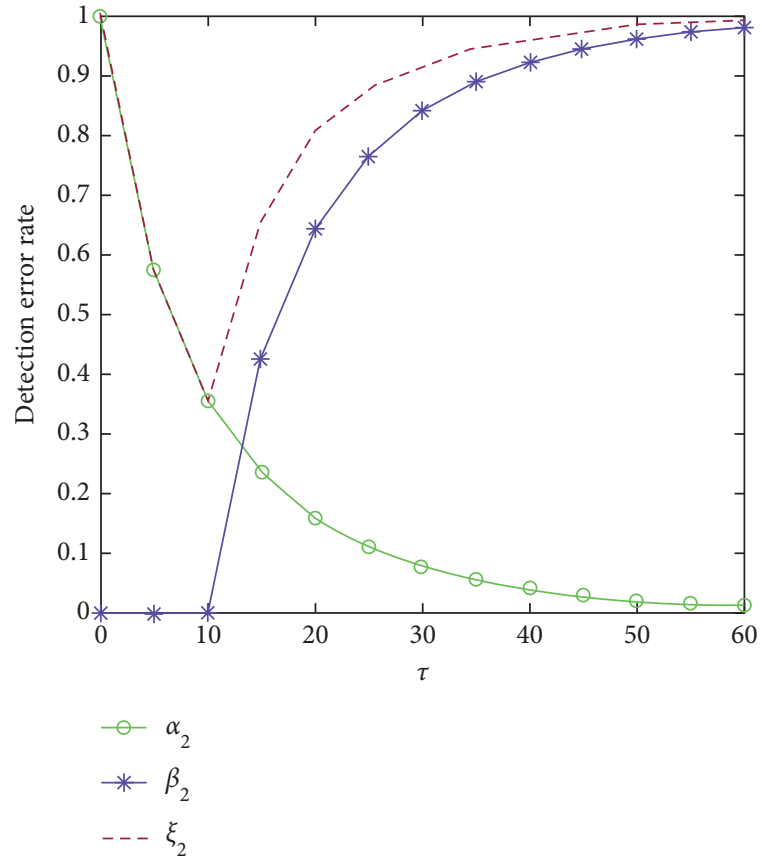

Figure 8: Minimum detection error rate, false alarm rate, and missed detection rate versus $\tau$, where $\sigma_{B}^{2}=0 d B, N_{R}=3$, $P_{R_{j}}^{\max }=P_{R_{k}}=10 \mathrm{~dB}$, and $\left|h_{R_{k} W}\right|^{2}=1$.

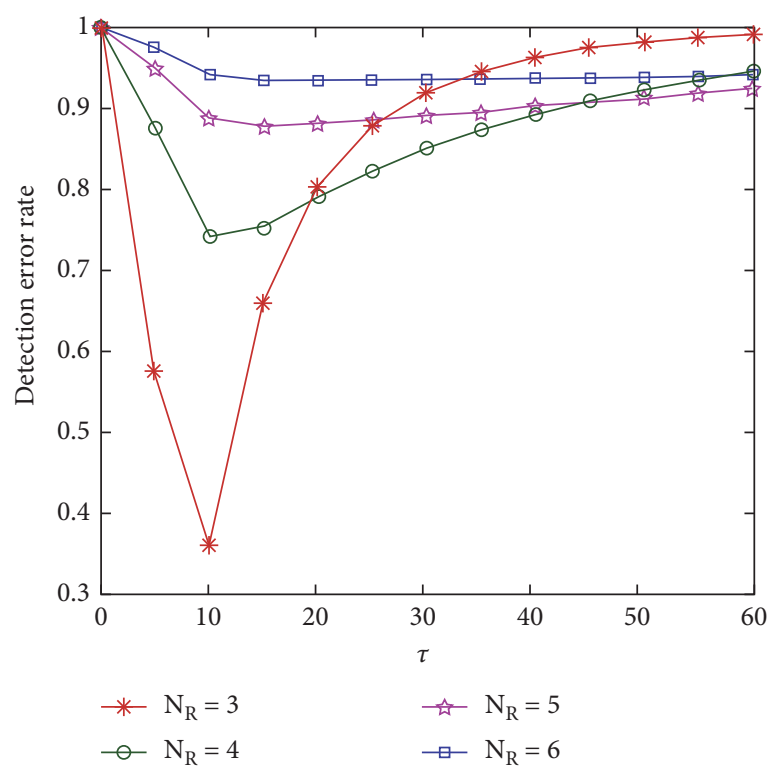

FIgURE 9: Minimum detection error rate versus $\tau$ with different value of $N_{R}$, where $\sigma_{B}^{2}=0 \mathrm{~dB}, \quad P_{R_{j}}^{\max }=P_{R_{k}}=10 \mathrm{~dB}$, and $\left|h_{R_{k} W}\right|^{2}=1$.

detection rate, and the larger the false alarm rate. It is difficult for Willie to detect the covert transmission with probability 1 , which is consistent with our expectation, and there is a minimum detection error probability; Figure 9 draws the detection error rate curve according to different antenna number $n$ and further illustrates the feasibility of minimum detection error rate under different parameters. This verifies the correctness of Theorem 5 . 
In Figures 10 and 11, the relationship between the minimum detection error probability $\xi_{2}$ and the maximum interference transmission power $P_{R_{j}}^{\max }$ of a single antenna formed by Relay using zero-forcing beamforming can be observed at the same time. The larger the $P_{R_{i}}^{\max }$ is, the greater the $\xi_{2}$ is. And Figure 10 also describes the relationship between the minimum detection error probability and the number of antennas $N_{R}$. It is easy to understand that the minimum detection error rate will increase with the increase in the number of antennas. This is because the larger the interference power and the number of antennas of a single antenna, the greater the total interference to Willie, and the greater the interference will hinder Willie's detection. Willie will be more prone to make errors, resulting in a higher error rate of Willie. This is consistent with the conclusion of Remark 3.1. In addition to describing the relationship between $\xi_{2}$ and $P_{R_{j}}^{\max }$, Figure 11 also plots the relationship between the minimum detection error probability $\xi_{2}$ and the power $P_{R_{K}}$ of Relay forwarding Alice covert messages. The larger the $P_{R_{K}}$ is, the smaller the $\xi_{2}$ is. This is because the higher the power of Relay forwarding covert messages is, the easier Willie is to detect the transmission of covert messages, and the smaller the probability of error is. This verifies the correctness of Remark 3.2.

6.2.2. Transmission Outage Probability. It can be seen from Figures 12 and 13 that the transmission outage probability $\delta_{2}$ is a monotonic decreasing function of Relay covert message transmission power $P_{R_{K}}$, that is, the larger the covert message transmission power $P_{R_{K}}$ is, the smaller the transmission interruption probability $\delta_{2}$ of the second stage is. This is because increasing $P_{R_{K}}$ will increase the SINR at Bob, so the more secure the transmission is, the less likely it is to be interrupted. This verifies the correctness of Remark 4.1. The relationship between the transmission outage probability $\delta_{2}$ and Bob's channel noise $\sigma_{B}^{2}$ is also described in Figure 12. $\delta_{2}$ increases with the increase of $\sigma_{B}^{2}$. This is because the increase of channel noise $e$ will reduce the signalto-interference-noise ratio at Bob, which makes the transmission easier to be interrupted. It can also be observed from Figure 12 that $\delta_{2}$ is a monotonic increasing function of $R$, that is, the higher the transmission rate is, the easier the transmission is interrupted. These observations are consistent with Remark 4.2. It can be observed from Figure 13 that the transmission outage probability $\delta_{2}$ is a monotonic decreasing function of the number of Relay antennas $N_{R}$, that is, the larger $N_{R}$ is, the smaller the transmission outage probability $\delta_{2}$ of the second stage is. This is because the increase in the number of antennas will increase the gain of the channel, thus making the transmission more secure. This verifies the correctness of Remark 4.3.

6.2.3. Maximum Effective Covert Rate. It can be seen from Figures 14 and 15 that the optimal effective covert rate $\overline{R_{C_{2}}^{*}}$ in the second stage is a monotonic increasing function of the interference transmission power $P_{R_{j}}^{\max }$ of a single Relay's antenna, that is, the greater the interference transmission power $P_{R_{j}}^{\max }$ of a single Relay's antenna, the greater the

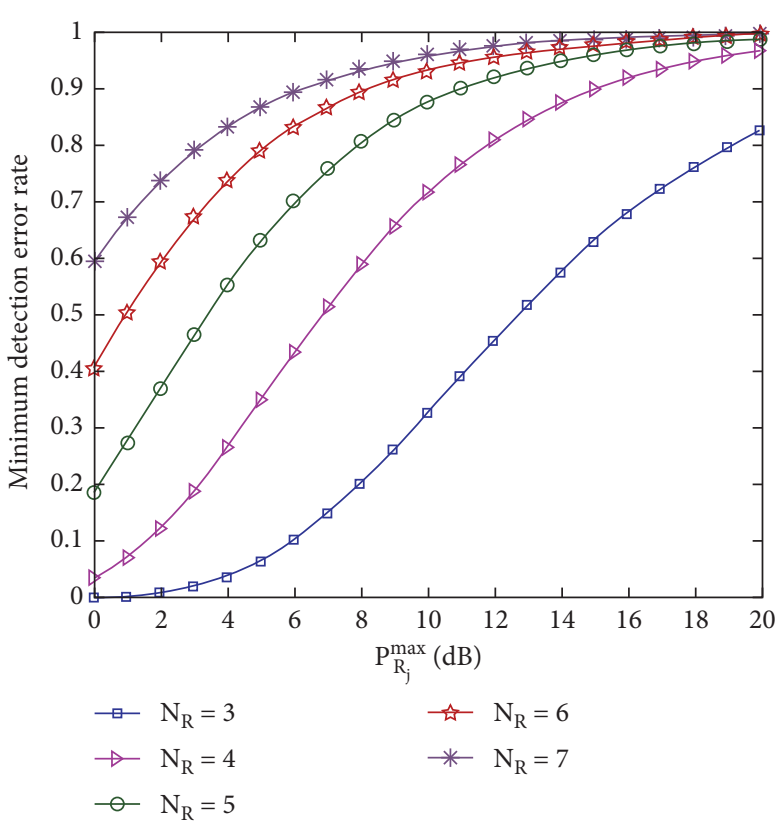

Figure 10: Minimum detection error rate versus $P_{R_{j}}^{\max }$ with different value of $N_{R}$, where $P_{R_{k}}=10 \mathrm{~dB}$ and $\left|h_{R_{k} W}\right|^{2}=1$.

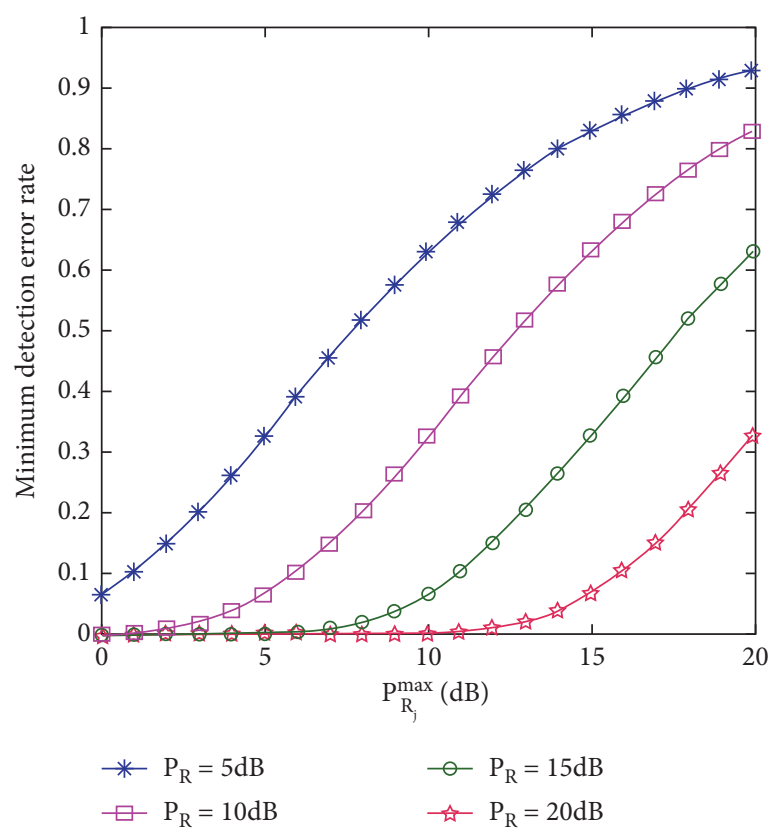

Figure 11: Minimum detection error rate versus $P_{R_{j}}^{\max }$ with different value of $P_{R_{k}}=10 \mathrm{~dB}$, where $N_{R}=3$ and $\left|h_{R_{k} W}\right|^{2}=1$.

optimal effective covert rate $\overline{R_{C_{2}}^{*}}$ in the second stage. This is because the greater the interference, the more likely Willie's detection is to make mistakes, and the higher the effective covert rate is. This is consistent with Remark 5.1. Similar to the first stage, the optimal effective covert rate $\overline{R_{C_{2}}^{*}}$ in the second stage increases with the increase of the number of antennas $N_{R}$ and $\varepsilon$. The reason has been described in the first stage, and it is not described here. This is consistent with the content of Remark 5.2. 


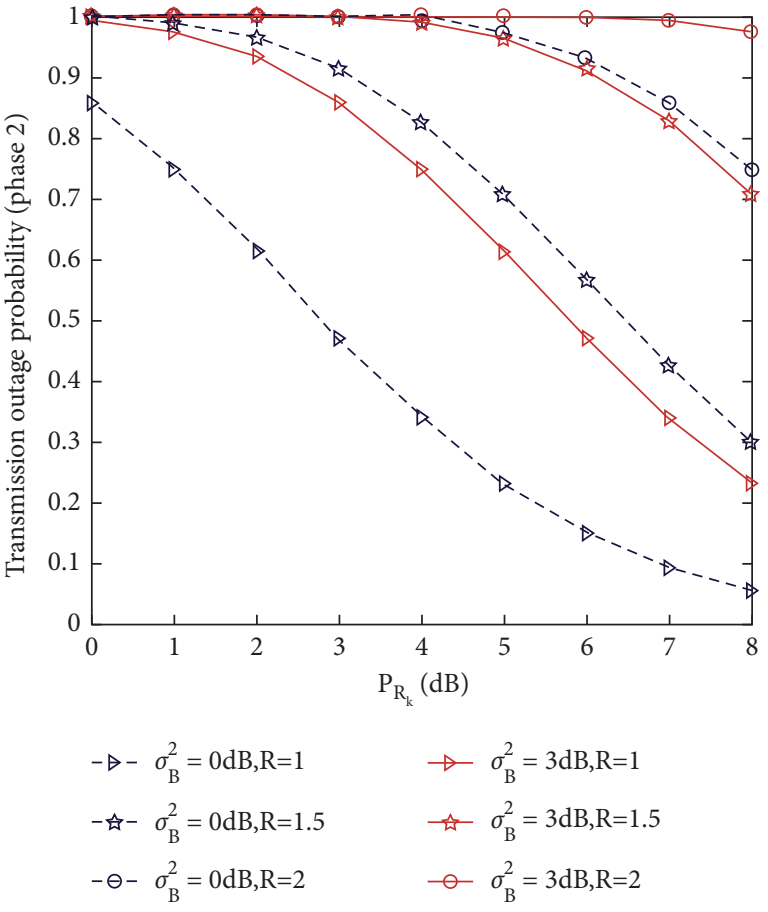

Figure 12: Transmission outage probability versus $P_{R_{k}}$ with different value of $\sigma_{B}^{2}$ and $R$, where $N_{R}=3$.

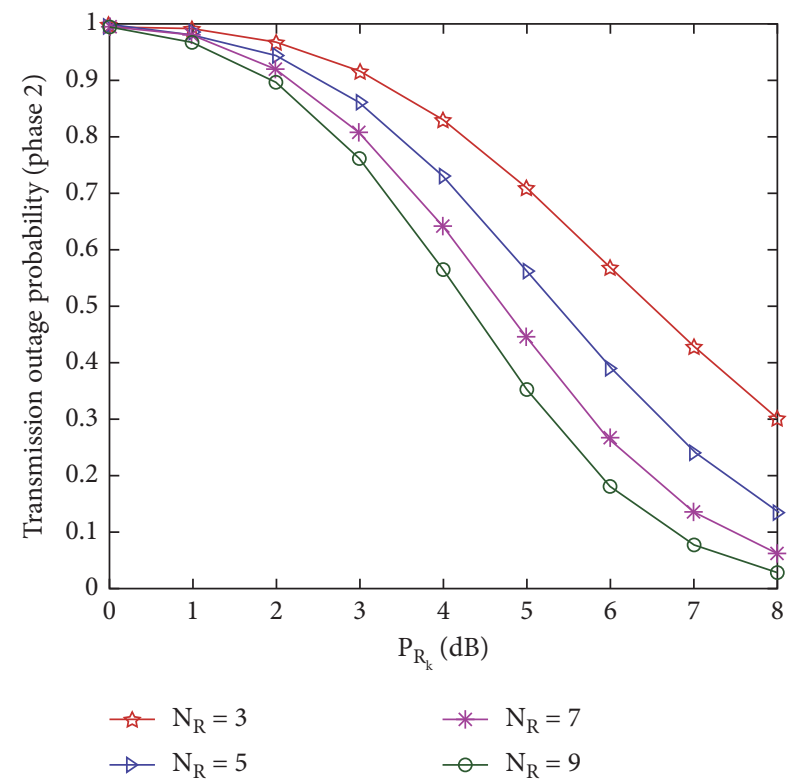

FIgURe 13: Transmission outage probability versus $P_{R_{k}}$ with different value of $N_{R}$, where $R=1.5$ and $\sigma_{B}^{2}=0 \mathrm{~dB}$.

Figure 16 shows the relationship between the optimal effective covert rate $\overline{R_{C_{2}}^{*}}$ and the power $P_{R_{K}}$ of Relay forwarding covert messages and the noise $\sigma_{B}^{2}$ of Bob channel. The higher the power $P_{R_{K}}$ of Relay forwarding covert messages, the higher the $\overline{R_{C_{2}}^{*}}$. The larger the noise $\sigma_{B}^{2}$ of Bob channel is, the smaller the $\overline{R_{C_{2}}^{*}}$ is. This is because the larger the $P_{R_{K}}$ is, the larger Bob's SINR is, which is the more conducive to the transmission of covert messages, the larger the $\sigma_{B}^{2}$ is, the smaller the SINR of Bob will

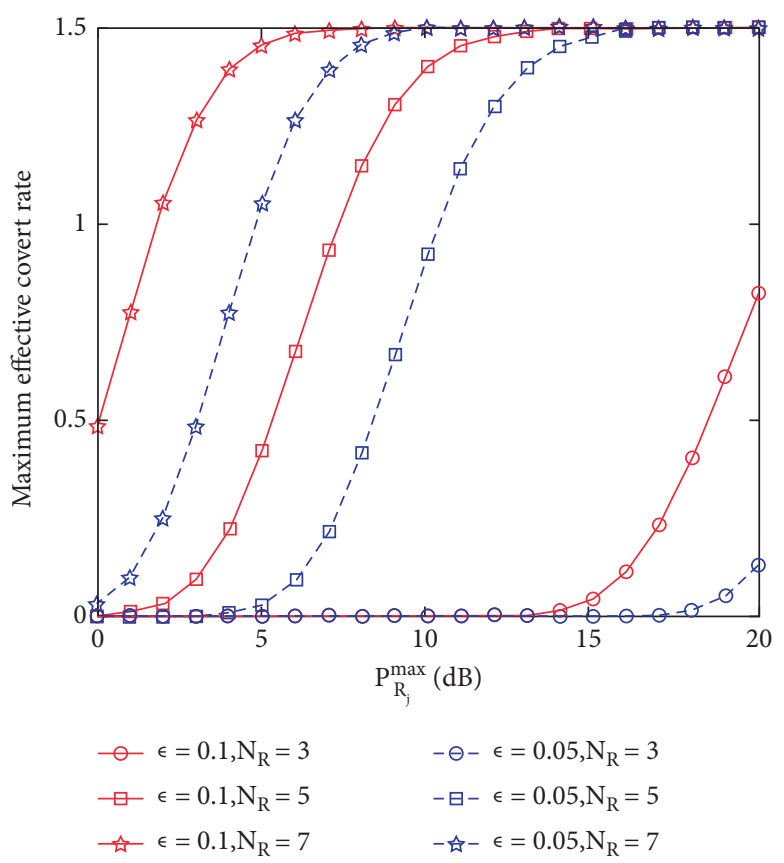

Figure 14: Maximum effective covert rate versus $P_{R_{\dot{j}}}^{\max }$ with different value of $N_{R}$ and $\varepsilon$, where $R=1.5,\left|h_{R_{j} W}\right|^{2}=1$, and $\sigma_{B}^{2}=0 d B$.

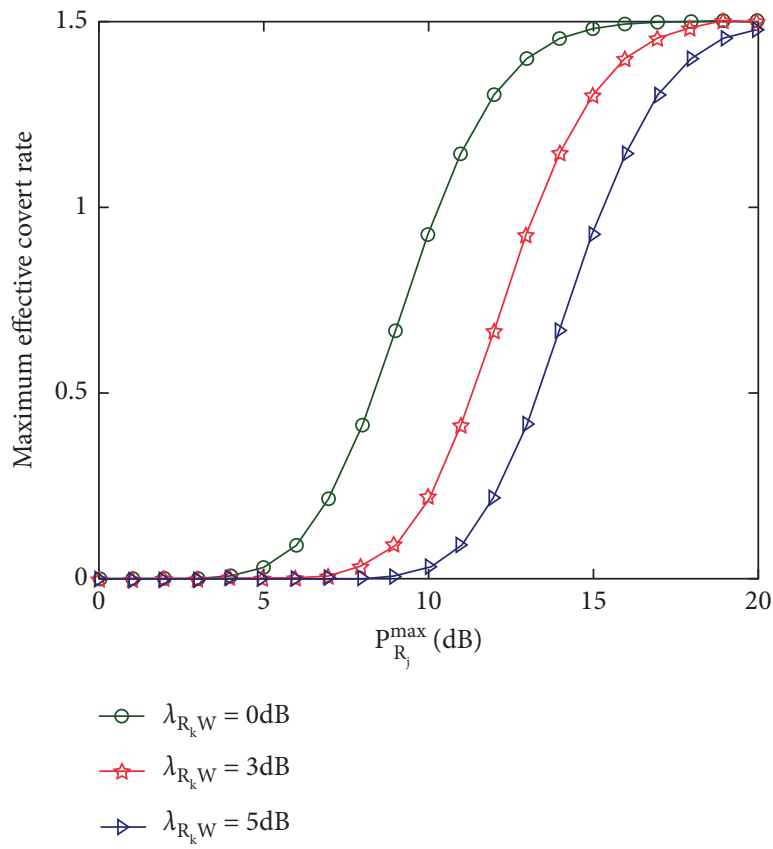

FIgURE 15: Maximum effective covert rate versus $P_{R_{k}}$ with different value of $\sigma_{B}^{2}$, where $R=1.5,\left|h_{R_{j} W}\right|^{2}=1, N_{R}=5$, and $\varepsilon=0.05$.

be, which is not conducive to covert message transmission. Figure 15 also describes the relationship between the optimal effective covert rate $\overline{R_{C_{2}}^{*}}$ and the channel coefficient $\left|h_{R_{k} W}\right|^{2}$. The larger the channel coefficient $\left|h_{R_{k} W}\right|^{2}$ is, the smaller the $\overline{R_{C_{2}}^{*}}$ is. Because it is the channel gain between Relay and Willie when Relay forwards Alice's covert messages, it is obvious that the 


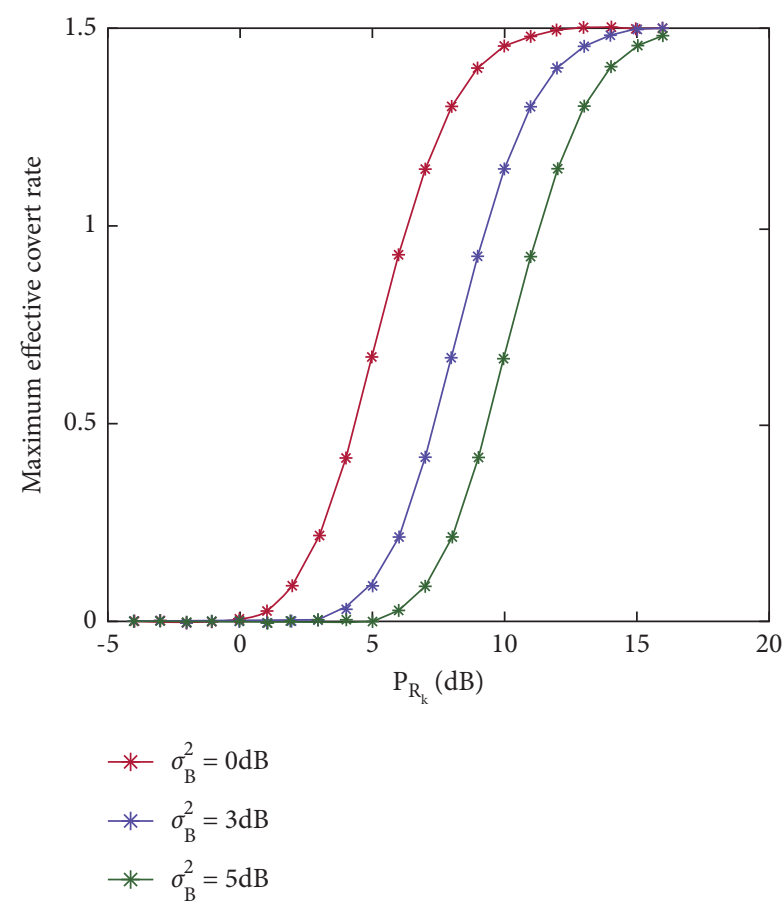

Figure 16: Maximum effective covert rate versus $P_{R_{j}}^{\max }$ with different value of $\left|h_{R_{j} W}\right|^{2}$, where $R=1.5, N_{R}=5, \varepsilon=0.05$, and $\sigma_{B}^{2}=0 \mathrm{~dB}$.

larger the coefficient is, the worse the transmission of covert messages will be. This is not only similar to the first stage conclusion but also verifies the correctness of Remark 5.3. In addition, Figures 14-16 shows that the effective covert rate of the second stage tends to a fixed value, which verifies the correctness of Corollary 2.

\subsection{Total Performance}

6.3.1. Transmission Outage Probability. As in the first and second stages, we also graphically plot the relevant parameters of the total transmission outage probability, as shown in Figures 17-20. By comprehensively comparing the transmission outage probability in the first and second stages, we can get the contents in Table 2. Among them, the upward front represents the increase of the value, and the downward arrow represents the decrease of the value.

It can be observed from Table 2 that for the same parameters, the first stage, the second stage, and the total transmission outage probability have the same conclusion, which is consistent with our expectation. The causes of these graph phenomena have been described in detail in the previous stages and will not be repeated here.

6.3.2. Maximum Effective Covert Rate. Because the total maximum effective covert rate is smaller in the first and second stages, the maximum effective covert rates of the first and second stages are drawn synthetically to facilitate the comparison. The details are shown in Figure 21. Obviously,

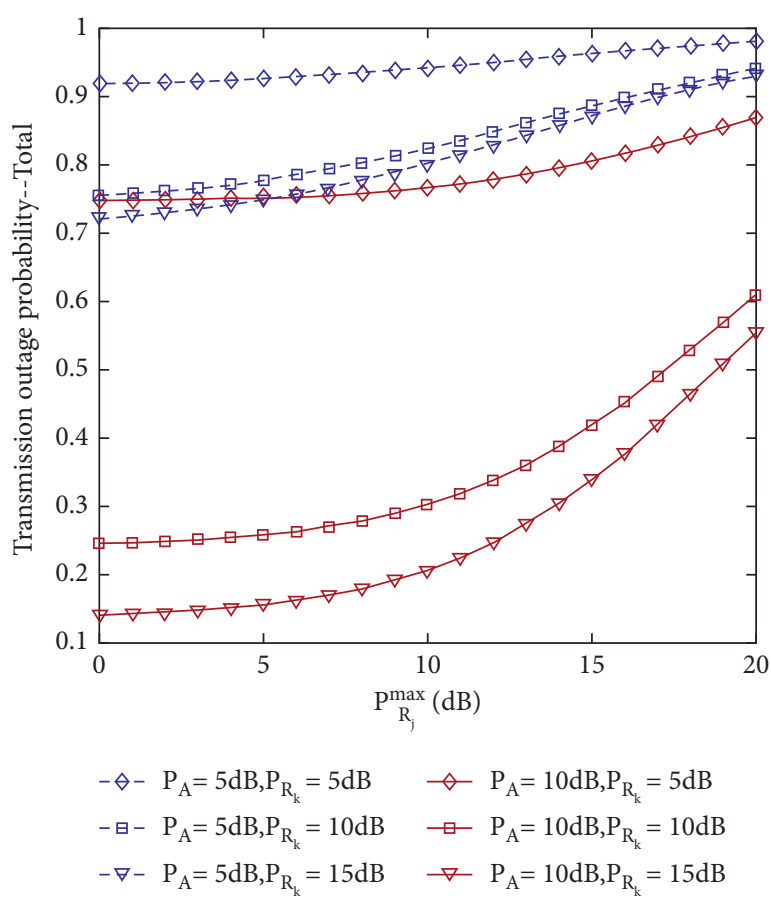

FIGURE 17: Total transmission outage probability versus $P_{R_{j}}^{\max }$ with different value of $P_{R_{k}}$ and $P_{A}$, where $\sigma_{R}^{2}=0 \mathrm{~dB}, \sigma_{B}^{2}=0 \mathrm{~dB}, R=1.5$, $N_{R}=3$, and $\phi=0.05$.

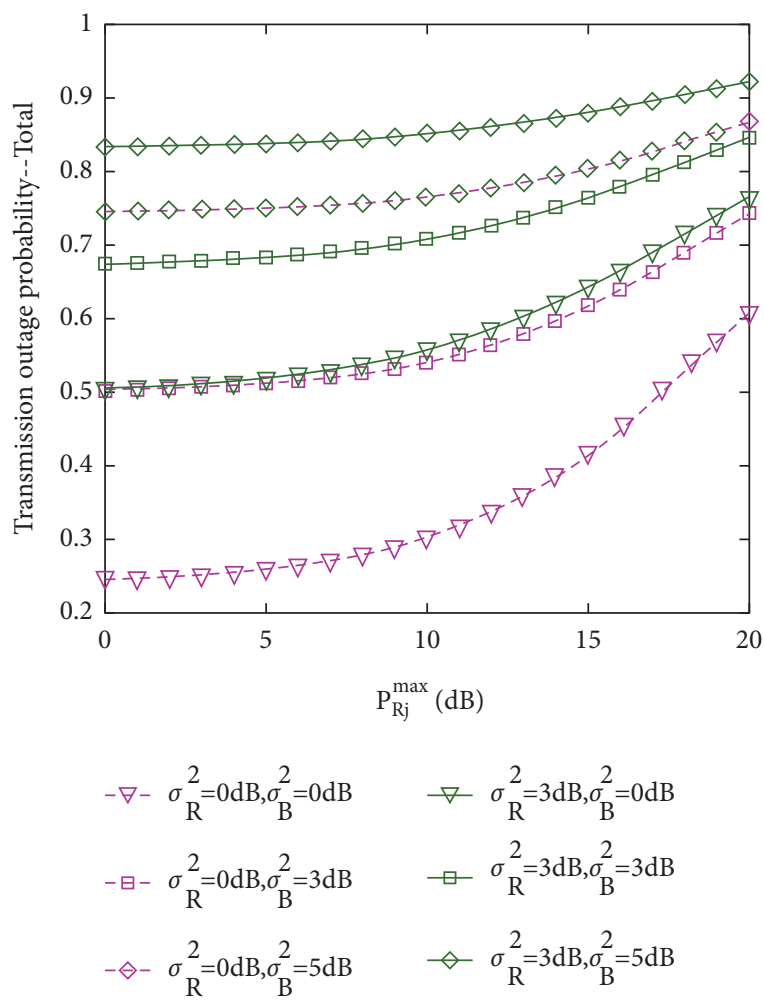

FIgURE 18: Total transmission outage probability versus $P_{R_{j}}^{\max }$ with different value of $\sigma_{R}^{2}$ and $\sigma_{B}^{2}$, where $P_{R_{k}}=10 \mathrm{~dB}, P_{A}=10 \mathrm{~dB}$, $R=1.5, N_{R}=3$, and $\phi=0.05$. 


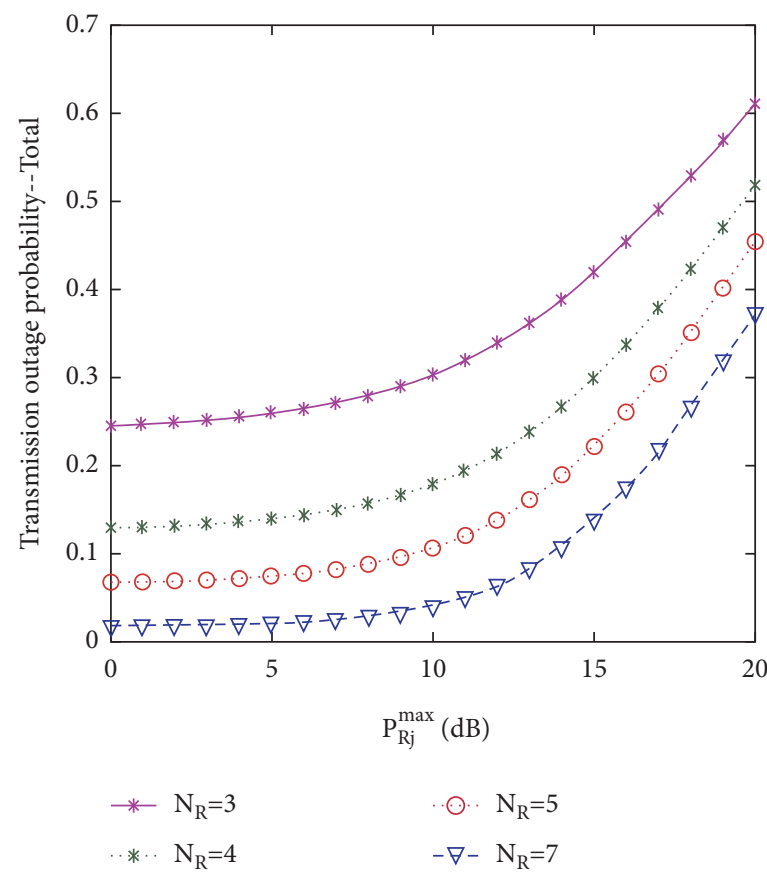

FIGURE 19: Total transmission outage probability versus $P_{R_{j}}^{\max }$ with different value of $N_{R}$, where $P_{R_{k}}=10 \mathrm{~dB}, P_{A}=10 \mathrm{~dB}, R=1.5, \phi=0.05$, $P_{R_{k}}=10 \mathrm{~dB}$, and $P_{A}=10 \mathrm{~dB}$.

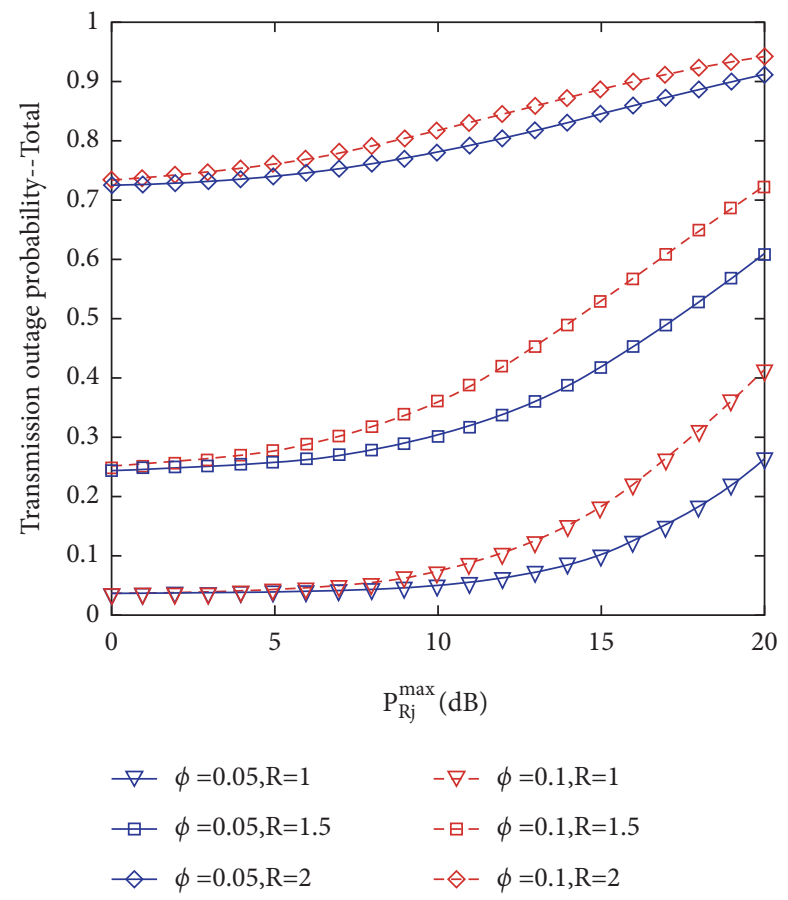

Figure 20: Total transmission outage probability versus $P_{R_{j}}^{\max }$ with different value of $\phi$ and $R$, where $P_{R_{k}}=10 d B, P_{A}=10 d B, N_{R}=3$, $\sigma_{R}^{2}=0 d B$, and $\sigma_{B}^{2}=0 d B$.

the maximum effective covert rate of the first stage is less than that of the second stage.

By comprehensively comparing the maximum effective covert rate in the first and second stages, we can get the contents in Table 3 .

It can be seen from Table 3 that for the same parameters, the total maximum effective concealment rate has the same conclusion as the maximum effective convert rate in one stage. This is because zero-forcing beamforming is used in the second stage to interfere with Willie without any impact on Bob, the transmission interruption probability will be smaller, and the corresponding maximum effective convert rate will be larger, so the maximum effective convert rate in the second stage is significantly higher than that in the first stage. This also verifies the correctness of Theorem 10 . 
TABLE 2: Transmission outage probability results.

\begin{tabular}{|c|c|c|c|c|}
\hline Parameter & Parameter variation & $\delta_{1}$ & $\delta_{2}$ & $\bar{\delta}$ \\
\hline Interference power & $\uparrow$ & $\uparrow$ & $\uparrow$ & $\uparrow$ \\
\hline Channel noise from the receiver & $\uparrow$ & $\uparrow$ & $\uparrow$ & $\uparrow$ \\
\hline Transmission rate & $\uparrow$ & $\uparrow$ & $\uparrow$ & $\uparrow$ \\
\hline Antennas number & $\uparrow$ & $\downarrow$ & $\downarrow$ & $\downarrow$ \\
\hline Covert message sending power & $\uparrow$ & $\downarrow$ & $\downarrow$ & $\downarrow$ \\
\hline Self-interference coefficient & $\uparrow$ & $\uparrow$ & - & $\uparrow$ \\
\hline
\end{tabular}

TABle 3: Maximum effective covert rate results.

\begin{tabular}{|c|c|c|c|c|}
\hline Parameter & Parameter variation & $\overline{R_{C_{1}}^{*}}$ & $\overline{R_{C_{2}}^{*}}$ & $\overline{R_{C}^{*}}$ \\
\hline Covert message sending power & $\uparrow$ & $\uparrow$ & $\uparrow$ & $\uparrow$ \\
\hline Covert constraints, $\varepsilon$ & $\uparrow$ & $\uparrow$ & $\uparrow$ & $\uparrow$ \\
\hline Antennas number & $\uparrow$ & $\uparrow$ & $\uparrow$ & $\uparrow$ \\
\hline Channel noise from the receiver & $\uparrow$ & $\downarrow$ & $\downarrow$ & $\downarrow$ \\
\hline Self-interference coefficient & $\uparrow$ & $\downarrow$ & - & $\downarrow$ \\
\hline The channel coefficient between covert message sender and Willie & $\uparrow$ & $\downarrow$ & $\downarrow$ & $\downarrow$ \\
\hline The channel coefficient between the AN sender and Willie & $\uparrow$ & $\uparrow$ & - & $\uparrow$ \\
\hline Interference power & $\uparrow$ & - & $\uparrow$ & - \\
\hline
\end{tabular}

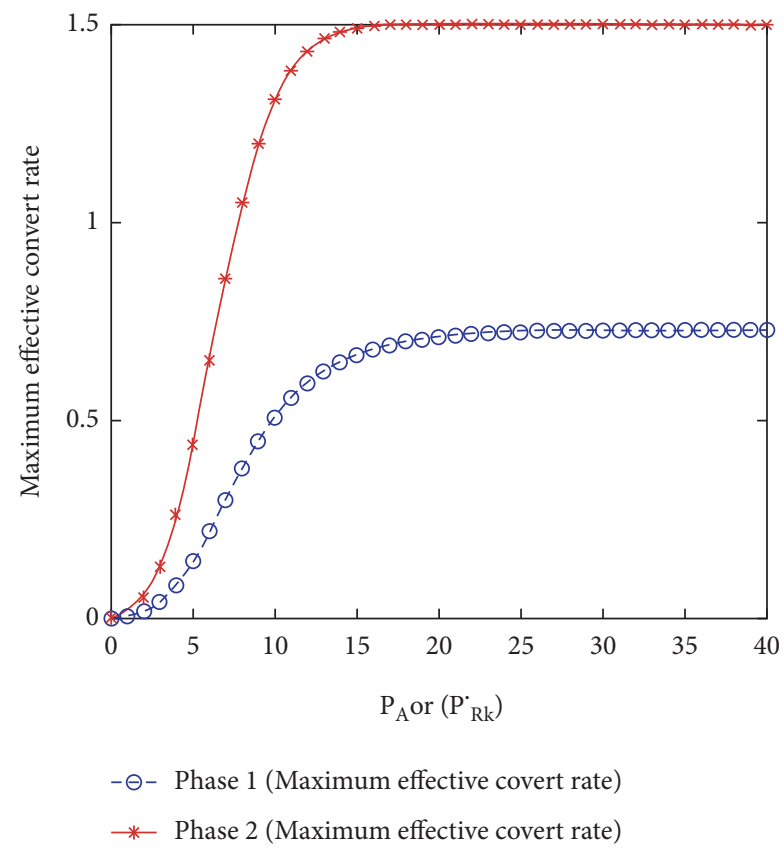

FIgURE 21: Comparison of stage 1 and stage 2 maximum effective covert rates, where $\sigma_{R}^{2}=0 d B, \sigma_{B}^{2}=0 d B, P_{R_{k}}=10 d B, R=1.5, \phi=0.01$, $N_{R}=3, \varepsilon=0.05,\left|h_{R_{j} W}\right|^{2}=1$, and $h_{A W}=1$.

\section{Conclusion}

In this work, we consider a wireless network covert communication system achieved by multiantenna full-duplex relay. The system is divided into two stages; each stage needs to complete the transmission of covert messages. In the first stage, Relay receives covert messages and sends interference to Willie. In the second stage, Relay decodes and forwards covert messages while interfering with Willie's detection.
The system must ensure that neither stage can be detected. The two stages and the total system performance are derived and analyzed. Under the constraint of covert transmission, the maximum effective covert rate of multiantenna single relay decode and forward relay network is studied, and the simulation results are analyzed in detail, the system can achieve excellent covert performance. In the future, multiantenna and multirelay wireless network covert communication systems can be further considered. 


\section{Appendix}

\section{A. Proof of Theorem 1}

When the channel capacity $C$ from Alice to Relay is less than the transmission rate $R$, the transmission from Alice to Relay will be interrupted. The channel capacity formula is as follows:

$$
C_{A R}=\frac{1}{2} \log _{2}\left(1+\gamma_{A R}\right) .
$$

In combination with formulas (1) and (A.1) and the definition of transmission interrupt probability, we have

$$
\begin{aligned}
\delta_{1} & =P\left\{\frac{P_{A} \max _{1 \leq i \leq N_{R}}\left(\left|h_{A R_{i}}\right|^{2}\right)}{\varphi P_{R_{j}}\left|h_{R_{j} R_{j}}\right|^{2}+\sigma_{R}^{2}}<2^{2 R}-1\right\}=\int_{0}^{P_{R_{j}}^{\max }} \int_{0}^{\infty}\left[1-\exp \left(-\frac{\left(2^{2 R}-1\right)\left(\varphi P_{R_{j}}\left|h_{R_{j} R_{j}}\right|^{2}+\sigma_{R}^{2}\right)}{P_{A}}\right)\right]^{N_{R}} \exp (-y) \frac{1}{P_{R_{j}}^{\max }} d x d y \\
& =1+\frac{P_{A}}{P_{R_{j}}^{\max } \varphi\left(2^{2 R}-1\right)} \sum_{m=1}^{N_{R}}\left(\begin{array}{c}
N_{R} \\
m
\end{array}\right)(-1)^{m} \frac{1}{m} \exp \left[-\frac{m\left(2^{2 R}-1\right) \sigma_{R}^{2}}{P_{A}}\right] \times \ln \left[1+m \varphi\left(2^{2 R}-1\right) \frac{P_{R_{j}}^{\max }}{P_{A}}\right]
\end{aligned}
$$

\section{B. Proof of Theorem 2}

According to formulas (11) and (13), $t_{\varepsilon}$ is the solution of $-t^{2}+t \ln t+1=1-\varepsilon$, and there is the definition of $t$ in formula (11), we have

$$
t_{\varepsilon}=\frac{P_{A}\left|h_{A W}\right|^{2}}{P_{A}\left|h_{A W}\right|^{2}+P_{R_{j}}^{\max }\left|h_{R_{j} W}\right|^{2}} .
$$

The proof can be completed by solving $P_{R_{j}}^{\max }$ in formula

\section{Proof of Theorem 3}

According to formula (13), we have

$$
\begin{aligned}
\overline{R_{C}}=R(1-\delta)=R\left\{\begin{array}{l}
1-1-\frac{P_{A}}{P_{R_{j}}^{\max } \varphi\left(2^{2 R}-1\right)} \sum_{m=1}^{N_{R}}\left(\begin{array}{c}
N_{R} \\
m
\end{array}\right)(-1)^{m} \frac{1}{m}, \\
\exp \left[-\frac{m\left(2^{2 R}-1\right) \sigma_{R}^{2}}{P_{A}}\right] \ln \left[1+m \varphi\left(2^{2 R}-1\right) \frac{P_{R_{j}}^{\max }}{P_{A}}\right],
\end{array}\right. \\
=-\frac{R P_{A}}{P_{R_{j}}^{\max } \varphi\left(2^{2 R}-1\right)} \sum_{m=1}^{N_{R}}\left(\begin{array}{c}
N_{R} \\
m
\end{array}\right)(-1)^{m} \frac{1}{m} \exp \left[-\frac{m\left(2^{2 R}-1\right) \sigma_{R}^{2}}{P_{A}}\right] \times \ln \left[1+m \varphi\left(2^{2 R}-1\right) \frac{P_{R_{j}}^{\max }}{P_{A}}\right] .
\end{aligned}
$$

By substituting formula (16) into formula (C.1), we can get the following results:

$$
\begin{aligned}
\overline{R_{C_{1}}^{*}} & =-R \sum_{m=1}^{N_{R}}\left(\begin{array}{c}
N_{R} \\
m
\end{array}\right)(-1)^{m} \exp \left[-\frac{m\left(2^{2 R}-1\right) \sigma_{R}^{2}}{P_{A}}\right] \times \frac{\ln \left[1+m \varphi\left(2^{2 R}-1\right)\left(1-t_{\varepsilon}\right)\left|h_{A W}\right|^{2} / t_{\varepsilon}\left|h_{R_{j} W}\right|^{2}\right]}{\left[m \varphi\left(2^{2 R}-1\right)\left(1-t_{\varepsilon}\right)\left|h_{A W}\right|^{2}\right] / t_{\varepsilon}\left|h_{R_{j} W}\right|^{2}}, \\
& =-R\left|h_{R_{j} W}\right|^{2} t_{\varepsilon} \sum_{m=1}^{N_{R}}\left(\begin{array}{c}
N_{R} \\
m
\end{array}\right)(-1)^{m} \exp \left[-\frac{m\left(2^{2 R}-1\right) \sigma_{R}^{2}}{P_{A}}\right] \times \frac{\ln \left[1+\left.m \varphi\left(2^{2 R}-1\right)\left(1-t_{\varepsilon}\right)\left|h_{A W}\right|^{2}\left|t_{\varepsilon}\right| h_{R_{j} W}\right|^{2}\right]}{m \varphi\left(2^{2 R}-1\right)\left(1-t_{\varepsilon}\right)\left|h_{A W}\right|^{2}} .
\end{aligned}
$$




\section{Proof of Theorem 4}

According to formulas (27) and (28), the false alarm rate is as follows:

$$
\begin{aligned}
\alpha_{2} & =P\left[P_{Z}\left|h_{R W}^{\dagger} W_{Z F}\right|^{2}+\sigma_{W}^{2}>\tau\right], \\
& =P\left[P_{Z}\left|h_{R W}^{\dagger} W_{Z F}\right|^{2}>\tau-\sigma_{W}^{2}\right], \\
& =P\left[\frac{P_{Z}\left|h_{R W}^{\dagger} W_{Z F}\right|^{2}}{\sigma_{W}^{2}}>\frac{\tau-\sigma_{W}^{2}}{\sigma_{W}^{2}}\right] .
\end{aligned}
$$

By using formula (23), we can get the result (D.2), shown at the top of the next page, where, by using the variable substitution of $P_{Z}=1 / x$, and with the help of [41], equation (3.351.2.11), and [41], equation (3.351.4), to solve the integral in formula (D.2), the result of formula (30) can be obtained as follows:

$$
\alpha_{2}=1-\int_{0}^{\left(N_{R}-1\right) P_{R_{j}}^{\max }}\left[1-\exp \left(-\frac{\tau-\sigma_{w}^{2}}{P_{Z}}\right) \sum_{l=0}^{N_{R}-3} \frac{1}{l !}\left(\frac{\tau-\sigma_{w}^{2}}{P_{Z}}\right)^{l}\right] \frac{1}{\left(N_{R}-1\right) P_{R_{j}}^{\max }} d P_{Z}
$$

In the same way, the miss detection rate is as follows:

$$
\begin{aligned}
\beta_{2} & =P\left[P_{R_{k}}\left|h_{R_{k} W}\right|^{2}+P_{Z}\left|h_{R W}^{\dagger} W_{Z F}\right|^{2}+\sigma_{W}^{2}<\tau\right], \\
& =P\left[P_{Z}\left|h_{R W}^{\dagger} W_{Z F}\right|^{2}<\tau-\sigma_{W}^{2}-P_{R_{k}}\left|h_{R_{k} W}\right|^{2}\right], \\
& =P\left[\frac{P_{Z}\left|h_{R W}^{\dagger} W_{Z F}\right|^{2}}{\sigma_{W}^{2}}<\frac{\tau-\sigma_{W}^{2}-P_{R_{k}}\left|h_{R_{k} W}\right|^{2}}{\sigma_{W}^{2}}\right] .
\end{aligned}
$$

By using (23), we can get the result (D.4), where by using the same variable substitution and solving the integral of (D.4) in the same way, the result of formula (31) can be obtained as follows:

$$
\beta_{2}=\int_{0}^{\left(N_{R}-1\right) P_{R_{j}}^{\max }}\left[1-\exp \left(-\frac{\tau-\sigma_{w}^{2}-P_{R_{k} W}\left|h_{R_{k} W}\right|^{2}}{P_{Z}}\right) \sum_{l=0}^{N_{R}-3} \frac{1}{l !}\left(\frac{\tau-\sigma_{w}^{2}-P_{R_{k} W}\left|h_{R_{k} W}\right|^{2}}{P_{Z}}\right)\right] \frac{1}{\left(N_{R}-1\right) P_{R_{j}}^{\max }} d P_{Z}
$$

\section{E. Proof of Theorem 5}

According to formulas (30) and (31), Willie's detection error rate (E.1) is shown at the top of the next page, where 


$$
\begin{aligned}
& x_{1}=\frac{\tau-\sigma_{w}^{2}}{\left(N_{R}-1\right) P_{R_{j}}^{\max }}, \\
& y_{1}=\frac{\tau-\sigma_{w}^{2}-P_{R_{k}}\left|h_{R_{k} W}\right|^{2}}{\left(N_{R}-1\right) P_{R_{j}}^{\max }},
\end{aligned}
$$

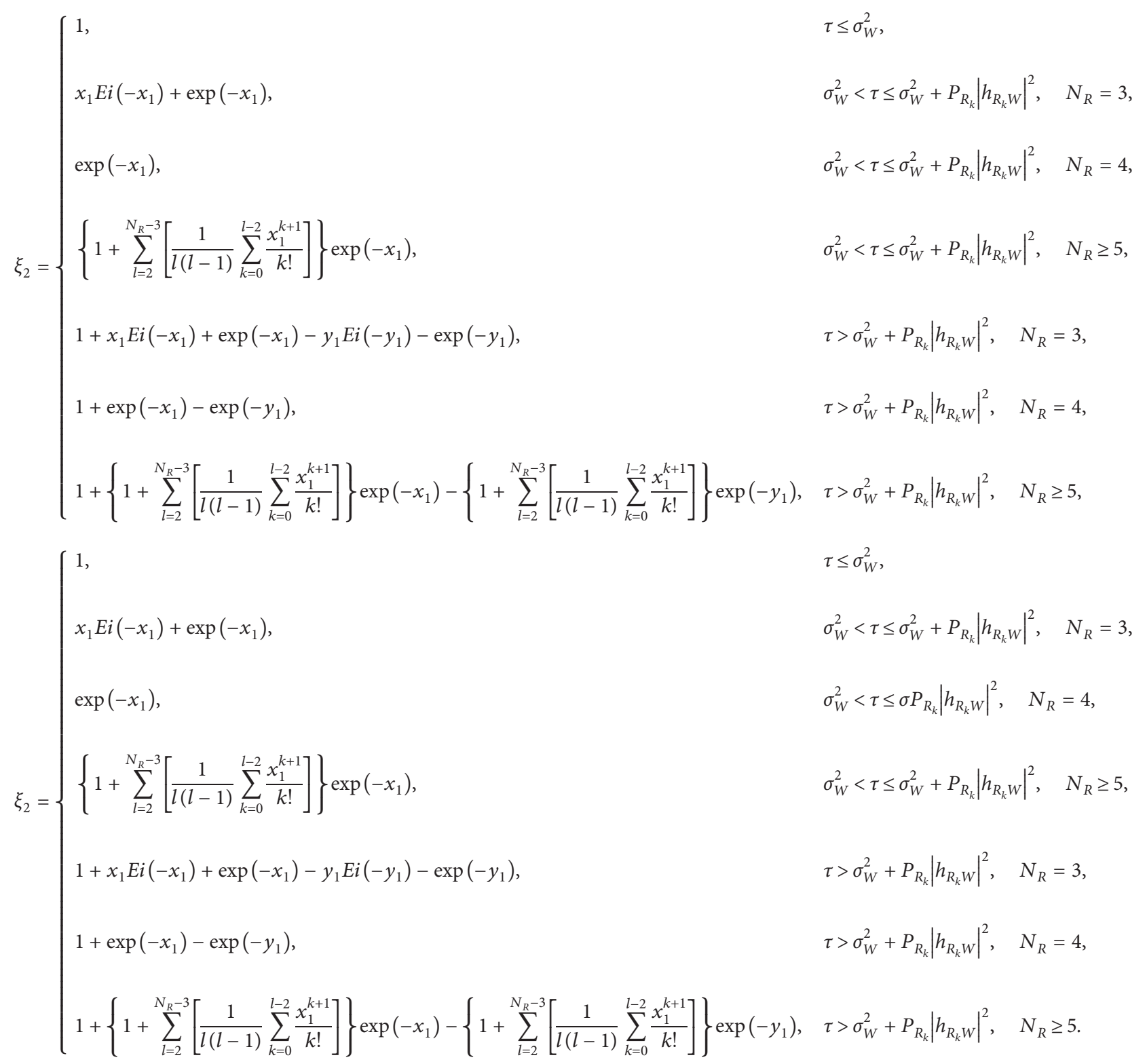

(1) When $N_{R}=3$, let us define $f(x)=x E i(-x)+\exp$ $(-x)$, then

$f^{\prime}(x)=E i(-x)<0 ; f^{\prime \prime}(x)=\frac{e^{-x}}{x}>0, \quad x>0$.

Therefore, the function $f(x)=x E i \quad(-x)+\exp$ $(-x)(x>0)$ decreases monotonically, and its first derivative increases monotonically.
When $\sigma_{W}^{2}<\tau \leq \sigma_{W}^{2}+P_{R_{k}}\left|h_{R_{k} W}\right|^{2}$, we have

$$
\frac{d \xi_{2}}{d \tau}=\frac{d f\left(x_{1}\right)}{d x_{1}} \times \frac{d x_{1}}{d \tau}=\frac{1}{\left(N_{R}-1\right) P_{R_{j}}^{\max }} \frac{d f\left(x_{1}\right)}{d x_{1}}<0 .
$$

When $\tau>\sigma_{W}^{2}+P_{R_{k}}\left|h_{R_{k} W}\right|^{2}$, we have 


$$
\frac{d \xi_{2}}{d \tau}=\frac{d f\left(x_{1}\right)}{d x_{1}} \times \frac{d x_{1}}{d \tau}-\frac{d f\left(y_{1}\right)}{d y_{1}} \times \frac{d y_{1}}{d \tau}=\frac{1}{\left(N_{R}-1\right) P_{R_{j}}^{\max }}\left[\frac{d f\left(x_{1}\right)}{d x_{1}}-\frac{d f\left(y_{1}\right)}{d y_{1}}\right]>0 .
$$

According to $x_{1}>y_{1}$ and the monotonicity of $f(x)=x E i(-x)+\exp (-x)(x>0)$, the $\min \xi$ is obtained at $\tau=\sigma_{W}^{2}+P_{R_{k}}\left|h_{R_{k} W}\right|^{2}$.

(2) Similarly, when $N_{R}=4$, let us define $g(x)=\exp (-x)$; the same derivation as $N_{R}=3$ is used. According to $x_{1}>y_{1}$ and the monotonicity of $g(x)$, the $\min \xi$ is obtained at $\tau=\sigma_{W}^{2}+P_{R_{k}}\left|h_{R_{k} W}\right|^{2}$.
(3) When $N_{R}=5$, the position transformation operation of the lower function $h(x)$ can be obtained as follows:

$$
h(x)=\left\{1+\sum_{l=2}^{N_{R}-3}\left[\frac{1}{l(l-1)} \sum_{k=0}^{l-2} \frac{x^{k+1}}{k !}\right]\right\} \exp (-x)=\left\{1+\sum_{k=1}^{N_{R}-4}\left[\sum_{l=k+1}^{N_{R}-3} \frac{1}{l(l-1)} \frac{x^{k}}{(k-1) !}\right]\right\} \exp (-x) .
$$

The first derivative can be expressed as follows:

$$
\begin{aligned}
h^{\prime}(x)=\left\{\begin{array}{l}
\sum_{k=1}^{N_{R}-4}\left[\sum_{l=k+1}^{N_{R}-3} \frac{1}{l(l-1)} \frac{k x^{k-1}}{(k-1) !}\right]-1 \\
-\sum_{k=1}^{N_{R}-4}\left[\sum_{l=k+1}^{N_{R}-3} \frac{1}{l(l-1)} \frac{x^{k}}{(k-1) !}\right] \exp (-x)
\end{array}\right. \\
=\left\{-\frac{1}{N_{R}-3}-\frac{1}{N_{R}-3} \sum_{k=1}^{N_{R}-4}\left[\frac{x^{k}}{k !}\right]\right\} \exp (-x)<0, x>0 .
\end{aligned}
$$

Its second derivative can be expressed as follows:

$$
\begin{aligned}
h^{\prime \prime}(x) & =\left\{\begin{array}{c}
-\frac{1}{N_{R}-3} \sum_{k=1}^{N_{R}-4}\left[\frac{x^{k-1}}{(k-1) !}\right] \\
+\frac{1}{N_{R}-3}+\frac{1}{N_{R}-3} \sum_{k=1}^{N_{R}-4}\left[\frac{x^{k}}{k !}\right]
\end{array}\right\} \exp (-x) \\
& =\frac{x^{N_{R}-4}}{\left(N_{R}-3\right) !} \exp (-x)>0, x>0 .
\end{aligned}
$$

Therefore, the function $h(x)$ is monotonically decreasing, and the first derivative is monotonically increasing.

When $\sigma_{W}^{2}<\tau \leq \sigma_{W}^{2}+P_{R_{k}}\left|h_{R_{k} W}\right|^{2}$, we have

$$
\frac{d \xi_{2}}{d \tau}=\frac{d h\left(x_{1}\right)}{d x_{1}} \times \frac{d x_{1}}{d \tau}=\frac{1}{\left(N_{R}-1\right) P_{R_{j}}^{\max }} \frac{d h\left(x_{1}\right)}{d x_{1}}<0
$$

When $\tau>\sigma_{W}^{2}+P_{R_{k}}\left|h_{R_{k} W}\right|^{2}$, we have

$$
\frac{d \xi_{2}}{d \tau}=\frac{d h\left(x_{1}\right)}{d x_{1}} \times \frac{d x_{1}}{d \tau}-\frac{d h\left(y_{1}\right)}{d y_{1}} \times \frac{d y_{1}}{d \tau}=\frac{1}{\left(N_{R}-1\right) P_{R_{j}}^{\max }}\left[\frac{d h\left(x_{1}\right)}{d x_{1}}-\frac{d h\left(y_{1}\right)}{d y_{1}}\right]>0
$$

According to $x_{1}>y_{1}$ and the monotonicity of $h(x)$, the $\min \xi$ is obtained at $\tau=\sigma_{W}^{2}+P_{R_{k}}\left|h_{R_{k} W}\right|^{2}$.
In summary, for any $N_{R} \geq 3$, there are the following conclusions. When $\sigma_{W}^{2}<\tau \leq \sigma_{W}^{2}+P_{R_{k}}\left|h_{R_{k} W}\right|^{2}$, there is 
$d \xi_{2} / d \tau=d \xi_{2} / d x d x / d \tau<0$. When $\tau>\sigma_{W}^{2}+P_{R_{k}}\left|h_{R_{k} W}\right|^{2}$, there is $d \xi_{2} / d \tau=d \xi_{2} / d x d x / d \tau-d \xi_{2} / d y d y / d \tau>0$.

Therefore, the optimal detection threshold is $\tau^{*}=\sigma_{w}^{2}+P_{R_{k} W}\left|h_{R_{k} W}\right|^{2}$, which can be substituted into formula (E.1) to obtain formula (33).

\section{F. Proof of Theorem 6}

When the channel capacity $C$ from Alice to Relay is less than the transmission rate $R$, the transmission from Relay to Bob will be interrupted. The channel capacity formula is as follows:

$$
C_{R B}=\frac{1}{2} \log _{2}\left(1+\gamma_{R B}\right) .
$$

In combination with formulas (19) and (F.1) and the definition of transmission interrupt probability, we have

$$
\delta=P\left\{\frac{P_{R_{k}} \max _{1 \leq k \leq N_{R}}\left|h_{R_{k} B}\right|^{2}}{\sigma_{B}^{2}}<2^{2 R}-1\right\}=P\left\{\max _{1 \leq k \leq N_{R}}\left|h_{R_{k} B}\right|^{2}<\frac{\left(2^{2 R}-1\right) \sigma_{B}^{2}}{P_{R_{k}}}\right\}=\left[1-\exp \left(-\frac{\left(2^{2 R}-1\right) \sigma_{B}^{2}}{P_{R_{k}}}\right)\right]^{N_{R}} .
$$

\section{G. Proof of Theorem 7}

According to the minimum detection error rate of (33) and its proof process, it is easy to get that for any $N_{R} \geq 3, \xi^{*}$ decreases monotonically with respect to $P_{R_{k}}$. So the optimal transmission power is determined by $\xi_{2}^{*}=1-\varepsilon$, and the combined formula (33) can get formula (38).

\section{H. Proof of Theorem 8}

According to formulas (35) and (37), we have

$$
\overline{R_{C}}=R(1-\delta)=R-R\left\{1-\exp \left[-\frac{\left(2^{2 R}-1\right) \sigma_{B}^{2}}{P_{R_{k}}}\right]\right\}^{N_{R}} .
$$

According to the remark of Theorem 6, the larger the $P_{R_{k}}$ is, the smaller the $\delta_{2}$ is, and the larger the $\overline{R_{C}}$ is. Therefore, $\overline{R_{C}}$ is a monotone increasing function of $P_{R_{k}}$. The maximum value of $\overline{R_{C}}$ should be the maximum $P_{R_{k}}$ satisfying the condition $\xi_{2}^{*} \geq 1-\varepsilon$, and for any $N_{R}>3$, $\xi^{*}$ decreases monotonically with respect to $P_{R_{k}}$, so the maximum $P_{R_{k}}$ satisfying condition $\xi_{2}^{*} \geq 1-\varepsilon$ is determined by $\xi_{2}^{*} \geq 1-\varepsilon$, that is, $P_{\mathrm{R}_{k}}^{*}$. By substituting formula (38) into formula (H.1), we can get formula (39).

\section{Proof of Theorem 9}

The transmission outage probability of the first stage is $\delta_{1}$, and the transmission outage probability of the second stage is $\delta_{2}$, so the transmission outage probability is given by

$$
\begin{aligned}
& \delta=1-\left(1-\delta_{1}\right)\left(1-\delta_{2}\right)=1-\left\{\begin{array}{c}
1-1+\frac{P_{A}}{P_{R_{j}}^{\max } \varphi\left(2^{2 R}-1\right)} \sum_{m=1}^{N_{R}}\left(\begin{array}{c}
N_{R} \\
m
\end{array}\right)(-1)^{m} \frac{1}{m} \\
\exp \left[-\frac{m\left(2^{2 R}-1\right) \sigma_{R}^{2}}{P_{A}}\right] \times \ln \left[1+m \varphi\left(2^{2 R}-1\right) \frac{P_{R_{j}}^{\max }}{P_{A}}\right]
\end{array}\right\} \times\left\{1-\left[1-\exp \left(-\frac{\left(2^{2 R}-1\right) \sigma_{B}^{2}}{P_{R_{k}}}\right)\right]^{N_{R}}\right\} . \\
& \min \left(\overline{R_{C_{1}}^{*}}, \overline{R_{C_{2}}^{*}}\right) .
\end{aligned}
$$

\section{J. Proof of Theorem 10}

The total optimal effective covert rate should be the minimum of the optimal effective covert rate in the first and second stages. results:

By changing formula (17), we can get the following

$$
\overline{R_{C_{1}}^{*}}=-R \sum_{m=1}^{N_{R}}\left(\begin{array}{c}
N_{R} \\
m
\end{array}\right)(-1)^{m} \exp \left[-\frac{m\left(2^{2 R}-1\right) \sigma_{R}^{2}}{P_{A}}\right] \times \frac{\ln \left[1+m \varphi\left(2^{2 R}-1\right)\left(1-t_{\varepsilon}\right)\left|h_{A W}\right|^{2} / t_{\varepsilon}\left|h_{R_{j} W}\right|^{2}\right]}{m \varphi\left(2^{2 R}-1\right)\left(1-t_{\varepsilon}\right)\left|h_{A W}\right|^{2} /\left(\left|h_{R_{j} W}\right|^{2} t_{\varepsilon}\right)} .
$$


By analyzing formula (J.2), we have

and

$$
\frac{\ln \left[1+m \varphi\left(2^{2 R}-1\right)\left(1-t_{\varepsilon}\right)\left|h_{A W}\right|^{2} / t_{\varepsilon}\left|h_{R_{j} W}\right|^{2}\right]}{m \varphi\left(2^{2 R}-1\right)\left(1-t_{\varepsilon}\right)\left|h_{A W}\right|^{2} /\left(\left|h_{R_{j} W}\right|^{2} t_{\varepsilon}\right)}<1
$$

$$
\sum_{m=1}^{N_{R}}\left(\begin{array}{c}
N_{R} \\
m
\end{array}\right)(-1)^{m} \exp \left[-\frac{m\left(2^{2 R}-1\right) \sigma_{R}^{2}}{P_{A}}\right] \times \frac{\ln \left[1+m \varphi\left(2^{2 R}-1\right)\left(1-t_{\varepsilon}\right)\left|h_{A W}\right|^{2} / t_{\varepsilon}\left|h_{R_{j} W}\right|^{2}\right]}{m \varphi\left(2^{2 R}-1\right)\left(1-t_{\varepsilon}\right)\left|h_{A W}\right|^{2} /\left(\left|h_{R_{j} W}\right|^{2} t_{\varepsilon}\right)}<0 .
$$

Therefore, formula (J.2) can be further derived as follows:

$$
\overline{R_{C_{1}}^{*}}<-R \sum_{m=1}^{N_{R}}\left(\begin{array}{c}
N_{R} \\
m
\end{array}\right)(-1)^{m} \exp \left[-\frac{m\left(2^{2 R}-1\right) \sigma_{R}^{2}}{P_{A}}\right]=R-R\left\{1-\exp \left[-\frac{\left(2^{2 R}-1\right) \sigma_{R}^{2}}{P_{A}}\right]\right\}^{N_{R}}
$$

Due to $\sigma_{R}^{2}=\sigma_{B}^{2}, P_{A}=P_{R_{k}}^{*}$, we have

$\overline{R_{C_{1}}^{*}}<R-R\left\{1-\exp \left[-\frac{\left(2^{2 R}-1\right) \sigma_{B}^{2}}{P_{R_{k}}^{*}}\right]\right\}^{N_{R}}={\overline{R_{C_{2}}}}^{*}$.

That is, $\min \left(\overline{R_{C_{1}}^{*}}, \overline{R_{C_{2}}^{*}}\right)=\overline{R_{C_{1}}^{*}}$.

\section{Data Availability}

Relevant data supporting the research results of this paper have been elaborated in this paper.

\section{Conflicts of Interest}

The authors declare that they have no conflicts of interest.

\section{Acknowledgments}

This work was supported by the National Natural Science Foundation of China under grant no. 62071486, the National Natural Science Foundation of Hunan Province under grant no. 2021JJ50051, the Scientific Research Project of Education Department Hunan Province, China (Nos. 19C0561 and 18C0531), the Natural Science Foundation on Frontier Leading Technology Basic Research Project of Jiangsu under grant no. BK2021200, and Key R \& D Program of Jiangsu Province (advanced research and key core technology on Industry) Key Project and Topics (BE2021095).

\section{References}

[1] M. Chernyshev, Z. Baig, O. Bello, and S. Zeadally, "Internet of things (IoT): research, simulators, and testbeds," IEEE Internet of Things Journal, vol. 5, no. 3, pp. 1637-1647, 2018.

[2] Y. Liu and H. Su, "Some necessary and sufficient conditions for containment of second-order multi-agent systems with sampled position data," Neurocomputing, vol. 378, no. 32, pp. 228-237, 2020.

[3] C. Xu, H. Xu, H. Su, and C. Liu, "Disturbance-observer based consensus of linear multi-agent systems with exogenous disturbance under intermittent communication," Neurocomputing, vol. 404, no. 3, pp. 26-33, 2020.

[4] Y. Liu and H. Su, "Containment control of second-order multi-agent systems via intermittent sampled position data communication," Applied Mathematics and Computation, vol. 362, no. 362, p. 124522, 2019.

[5] C. Xu, B. Li, and L. Yang, "Semi-global containment of discrete-time high-order multi-agent systems with input saturation via intermittent control," IET Control Theory \& Applications, vol. 14, no. 16, pp. 2303-2309, 2020.

[6] Z. Xiang, W. Yang, Y. Cai, Z. Ding, and Y. Song, "Secure transmission design in HARQ assisted cognitive NOMA networks," IEEE Transactions on Information Forensics and Security, vol. 15, pp. 36-43, 2020.

[7] T. Zgang, Y. Huang, Y. Cai, C. Zhong, Y. Yang, and K. George, "Secure multi-antenna cognitive wiretap networks," IEEE Transactions on Vehicular Technology, vol. 66, pp. 36-43, 2017.

[8] X. Sun, W. Yang, Y. Cai, Z. Xiang, and X. Tang, "Secure transmissions in millimeter wave SWIPT UAV-based relay networks," IEEE Wireless Communications Letters, vol. 8, no. 3, pp. 785-788, 2019.

[9] X. Lu, W. Yang, X. Guan, and Y. Cai, "DCE-based secure transmission for massive MIMO relay system against active eavesdropper," IEEE Transactions on Vehicular Technology, vol. 69, no. 11, pp. 13045-13059, 2020.

[10] Z. Xiang, W. Yang, G. Pan, Y. Cai, Y. Song, and Y. Zou, "Secure transmission in HARQ-assisted non-orthogonal multiple access networks," IEEE Transactions on Information Forensics and Security, vol. 15, pp. 20-25, 2020.

[11] X. Lu, W. Yang, X. Guan, Q. Wu, and Y. Cai, "Robust and secure beamforming for intelligent reflecting surface aided mmWave MISO systems," IEEE Wireless Communications Letters, vol. 9, no. 12, pp. 2068-2072, 2020. 
[12] Y. Wang, S. Yan, W. Yang, and Y. Cai, "Covert communications with constrained age of information," IEEE Wireless Communications Letters, vol. 10, no. 2, pp. 368-372, 2021.

[13] L. Tao, W. Yang, S. Yan, D. Wu, X. Guan, and D. Chen, "Covert communication in downlink NOMA systems with random transmit power," IEEE Wireless Communications Letters, vol. 9, no. 11, pp. 2000-2004, 2020.

[14] L. Yang, W. Yang, S. Xu, L. Tang, and Z. He, "Achieving covert wireless communications using a full-duplex multi-antenna receiver," in Proceedings of the International Conference on Computer and Communications (ICCC), IEEE, Chengdu, China, Dec. 2019.

[15] B. A. Bash, D. Goeckel, and D. Towsley, "Limits of reliable communication with low probability of detection on AWGN channels," IEEE Journal on Selected Areas in Communications, vol. 31, no. 9, pp. 1921-1930, 2013.

[16] A. Abdelaziz and C. E. Koksal, "Fundamental limits of covert communication over MIMO AWGN channel," Proc. IEEE Conf. Commun. Network Security (CNS), vol. 31, no. 9, pp. 1-9, 2017.

[17] L. Wang, G. W. Wornell, and L. Zheng, "Fundamental limits of communication with low probability of detection," IEEE Transactions on Information Theory, vol. 62, no. 6, pp. 3493-3503, 2016.

[18] K. S. K. Arumugam and M. R. Bloch, "Keyless covert communication over multiple-access channels," Proc. IEEE Int. Symp. Inf. Theory (ISIT), vol. 62, no. 6, pp. 2229-2233, 2016.

[19] P. H. Che, M. Bakshi, and S. Jaggi, "Reliable deniable communication: hiding messages in noise," Proc. IEEE Int. Symp. Inf. Theory, vol. 13, no. 9, pp. 2945-2949, 2013.

[20] P. Mukherjee and S. Ulukus, "Covert bits through queues," Proc. IEEE Conf. Commun. Netw. Security (CNS), vol. 60, no. 17, pp. 626-630, 2016.

[21] Z. Liu, J. Liu, Y. Zeng, and J. Ma, “Covert wireless communications in IoT systems:hiding information in interference," IEEE Wireless Communications, vol. 18, no. 33, pp. 46-52, 2018.

[22] G. Shabsigh and V. Frost, "Stochastic geometry for the analysis of wireless covert networks," Milcom Track five-Selected Topics in Communications, vol. 18, no. 33, pp. 46-52, 2016.

[23] T. G. Dvorkind and A. Cohen, "Maximizing miss detection for covert communication under practical constraints," IEEE Workshop on Statistical Signal Processin, vol. 18, no. 33, pp. 46-52, 2018.

[24] F. Shu, T. Xu, J. Hu, and S. Yan, "Delay-constrained covert communications with a full-duplex receiver," IEEE Wireless Communications Letters, vol. 8, no. 3, pp. 813-816, 2019.

[25] J. Wang, W. Tang, Q. Zhu, X. Li, H. Rao, and S. Li, "Covert communication with the help of relay and channel uncertainty," IEEE Wireless Communications Letters, vol. 8, no. 1, pp. 317-320, 2019.

[26] K. Shahzad, X. Zhou, S. Yan, J. Hu, F. Shu, and J. Li, "Achieving covert wireless communications using a full-duplex receiver," IEEE Transactions on Wireless Communications, vol. 17, no. 12, pp. 8517-8530, 2018.

[27] J. Hu, S. Yan, X. Zhou, F. Shu, J. Li, and J. Wang, "Covert communication achieved by a greedy relay in wireless networks," IEEE Transactions on Wireless Communications, vol. 17, no. 7, pp. 4766-4779, 2017.

[28] J. Hu, S. Yan, F. Shu, and J. Wang, "Covert transmission with a self-sustained relay," IEEE Transactions on Wireless Communications, vol. 18, no. 18, pp. 4089-4102, 2019.
[29] M. Forouzesh, P. Azmi, A. Kuhestani, and P. L. Yeoh, "Covert communication and secure transmission over untrusted relaying networks in the presence of multiple wardens," IEEE Transactions on Communications, vol. 68, no. 6, pp. 37373749, 2020.

[30] A. Sheikholeslami, M. Ghaderi, D. Towsley, B. A. Bash, S. Guha, and D. Goeckel, "Multi-hop routing in covert wireless networks," IEEE Transactions on Wireless Communications, vol. 17, no. 6, pp. 3656-3669, 2018.

[31] H.-M. Wang, Y. Zhang, X. Zhang, and Z. Li, "Secrecy and covert communications against UAV surveillance via multihop networks," IEEE Transactions on Communications, vol. 68, no. 1, pp. 389-401, 2020.

[32] X. Lu, W. Yang, Y. Cai, and X. Guan, "Proactive eavesdropping via covert pilot spoofing attack in multi-antenna systems," IEEE Access, vol. 7, 2019.

[33] Y. Li, R. Zhao, Y. Deng, F. Shu, and Z. Nie, "Harvest-andOpportunistically-Relay: analyses on transmission outage and covertness," IEEE Transactions on Wireless Communications, vol. 97, no. 17, pp. 148-162, Aug. 2020.

[34] F. Shu, T. Xu, J. Hu, and S. Yan, "Delay-Constrained covert communications with a full-duplex receiver," IEEE Transactions on Wireless Communications, vol. 68, no. 1, pp. 469485, 2019.

[35] T. Zheng, L. Xu, X. Liu, and Z. Lu, "“'Covert communication with A full-duplex Receiver based on channel distribution information," international symposium on antennas," International Symposium on An-tennas, vol. 798, no. 13, pp. 3891-3899, 2019.

[36] O. Shmuel, A. Cohen, O. Gurewitz, and A. Cohen, "Multiantenna Jamming in covert Communication," in Proceedings of the 2019 IEEE International Symposium on Information Theory (ISIT), IEEE, Paris, France, July 2019.

[37] J. Hu, K. Shahzad, S. Yan, X. Zhou, F. Shu, and J. Li, "Covert Communications with a Full-Duplex Receiver over Wireless Fading Channels," in Proceedings of the IEEE Int. Conf. Commun. (ICC), pp. 1-6, IEEE, Kansas City, MO, USA, May 2018.

[38] J. Hu, S. Yan, X. Zhou, F. Shu, and J. Wang, "Covert Communication in Wireless Relay Networks," in Proceedings of the IEEE Global Communications Conference, IEEE, Singapore, December 2017.

[39] E. Lehmann and J. Romano, Testing Statistical Hypotheses, Springer Press, New York, NY, USA, 3rd edition, 2005.

[40] Basilevsky, Applied Matrix Algebra in the Statistical Sciences, North-Holland, New York, 1983.

[41] A. Afana, V. Asghari, A. Ghrayeb, and S. Affes, "Cooperative relaying in spectrum-sharing systems with beamforming and interference constraints," in Proceedings of the 2012 IEEE 13th International Workshop on Signal Processing Advances in Wireless Communications (SPAWC), IEEE, Cesme, Turkey, June 2012.

[42] L. Yang, W. Yang, J. Tu, X. Lu, L. Tang, and Z. He, "Covert communication achieved by a full-duplex multi-antenna receiver in wireless networks," Journal of Circuits, Systems, and Computers, p. 2150258, 2021. 